\title{
Investigating a simple model of cutaneous wound healing angiogenesis
}

Received: 24 August 2001 / Revised version: 18 April 2002 /

Published online: 23 August 2002 - (C) Springer-Verlag 2002

\begin{abstract}
A simple model of wound healing angiogenesis is presented, and investigated using numerical and asymptotic techniques. The model captures many key qualitative features of the wound healing angiogenic response, such as the propagation of a structural unit into the wound centre. A detailed perturbative study is pursued, and is shown to capture all features of the model. This enables one to show that the level of the angiogenic response predicted by the model is governed to a good approximation by a small number of parameter groupings. Further investigation leads to predictions concerning how one should select between potential optimal means of stimulating cell proliferation in order to increase the level of the angiogenic response.
\end{abstract}

\section{Introduction and biological background}

Cutaneous wound healing has developed in higher organisms over the course of evolution, resulting in complex mechanisms for the intricate control and orchestration of cells, soluble factors and extra-cellular matrix following integument injury. The outer layer of the skin, the epidermis, lies on a basal lamina covering the dermis, which is a matrix of collageneous fibres interwoven with blood vessels, lymphatic vessels and nerves, and contains numerous free cells, such as fibroblasts, lymphocytes and mast cells.

Dermal repair consists of three overlapping phases: inflammation, proliferation and remodelling. The inflammatory phase encompasses blood clot formation and immune system response. Regeneration of the epidermis also begins during this period, within hours after injury [3]. During the proliferative phase cells and intercellular substances increase greatly, accompanied by the migration of macrophages, fibroblasts and blood capillaries across the wound bed. This migration is observed to occur in a structural unit, often referred to as a wound module [16] or healing unit [1], consisting of activated macrophages in the lead, followed by fibroblasts, then by capillary tips and, finally, capillaries. The macrophages, recruited into the hypoxic wound bed, release chemotactic agents, continually attracting the

E.A. Gaffney: The School of Mathematics and Statistics, The University of Birmingham, Edgbaston, Birmingham B15 2TT, U.K. e-mail: eag@for . mat . bham.ac . uk

K. Pugh, P.K. Maini: Centre for Mathematical Biology, Mathematical Institute, 24-29 St Giles', Oxford OX1 3LB U.K.

F. Arnold: John Radcliffe Hospital, Oxford OX3 9DU, U.K.

Key words or phrases: Angiogenesis - Wound Healing - Travelling Waves - Asymptotics 
fibroblasts and capillaries into the wound. The fibroblasts in turn produce an extracellular matrix which facilitates further cell migration into the wound and provides mechanical support for the new capillaries. The newly formed capillaries, in turn, supply nutrients, enabling continued cellular function within the structural unit, which consequently migrates across the wound due to the mutual interaction of its constituents [16]. The tissue formed in the wound as a result of the structural unit's migration is capillary and cell rich, and referred to as granulation tissue.

The final phase, remodelling, involves wound contraction; the maturation of the wound bed tissue, which gradually evolves from a highly cellular and vascularised state to form scar tissue, with few cells and blood vessels; and the maturation of the extra-cellular matrix, resulting in higher tensile strength and a less random orientation of the collageneous fibres.

We briefly note that this relatively elegant picture is in fact far from complete. Many other mechanisms are at work, as one has to expect from a real biological system. In addition to chemotaxis and hypoxia, numerous signals produced by the macrophages and surrounding tissue influence the wound healing system. For example growth and chemokinetic factors are also observed to be present [17] as is macrophage secreted lactate, which upregulates fibroblast collagen synthesis [16]. Furthermore, there is the possibility of numerous interactions between these various signals; to illustrate, experiments have indicated that the production of macrophage factors is controlled by oxygen levels [1]. To attempt to incorporate such detail is inappropriate in a modelling study, and hence below we focus on the fundamental, if basic, idea of a structural unit invading the wound bed, and even this framework is considered in a simplified form to facilitate modelling.

Of central interest in this paper is the mechanism for new capillary formation, or angiogenesis, within the structural unit, and we proceed to describe this subprocess in detail. Consider the structure of a blood capillary near the wound edge. Aside from the blood constituents it carries, the capillary is a cylinder of endothelial cells, enveloped by a basement membrane. Angiogenesis begins with the degradation of the basement membrane at localised sites which face the wound. This is followed by the migration of endothelial cells out of the vessel, aligning so as to form a tube, or "sprout", which extends from the parent vessel. Cells behind the newly formed "capillary tips" proliferate, extending the sprouts further in the direction of the wound. These tips can branch and eventually join up with other sprouts (anastomosis) to form a complete circuit through which blood can flow. The sprouting process begins again from these new vessels, until the wound space is permeated by a network of new capillaries (see Figure 1), covered first by a provisional layer and later a true basement membrane, both synthesized by the cells of the capillaries [3, pages 18-19].

Many aspects, though certainly not all, of the picture of dermal wound healing described above have been the subject of modelling investigations, including collagen fibre formation and orientation [4], and wound contraction [8]. In particular, there are a number of models for dermal wound healing angiogenesis (DWHA) in the literature $[2,10,13,14]$. These works involve developing relatively complex models, which are investigated primarily using numerical techniques with the aim of firstly achieving agreement with experimental observation and, 


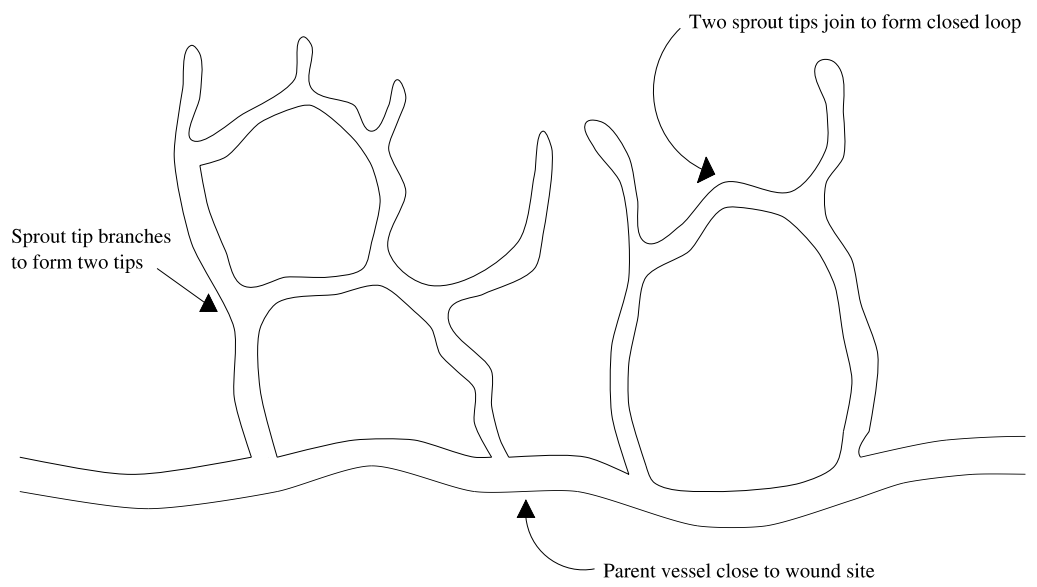

Fig. 1. Sprout tips branch and join up to form a capillary network

secondly, acquiring insight into the important factors and parameters influencing DWHA.

For example Pettet et al. [14] develop a six variable model incorporating densities of capillary tips, blood vessels, fibroblasts, macrophage-derived chemoattractants, oxygen and extracellular matrix, and it is shown that numerical simulations produce results in qualitative agreement with experimental observations. Other papers consider further simplified systems. Olsen et al. [10] consider a two variable model to investigate how the extracellular matrix density influences endothelial cell densities. Models incorporating capillary tips, blood vessels and macrophagederived chemoattractants are considered in $[2,13]$. In the former paper, the behaviour of front propagation speeds are compared to experimentally observed wound healing speeds. In the latter paper, the numerics is supplemented with an analysis investigating bounds on both the travelling wavespeeds and healed blood vessel densities.

The focus for this paper concerns a minimalist, but certainly non-trivial, model of DWHA phenomenology in terms of only two model constituents, namely densities of capillary tips and endothelial cells. We show that the model constructed in this paper displays the qualitative features of the structural unit observed empirically. Straightforward estimates for wound healing speeds are deduced, as is perfectly possible with previous models. A key aspect of this model is that other features of the model's structural unit are amenable to detailed analytical investigation. This is used to demonstrate the non-intuitive result that the maximum blood vessel density and the maximum density of "capillary tips" (at least as predicated by the model) depend on a very limited number of parameter groupings to a good approximation and such insight is confirmed numerically.

One may interpret maximal blood vessel densities as a measure of the system's angiogenic ability and its ability to supply nutrients to the wound healing structural unit. Consequently, the prediction that such ability should be regulated by a small number of parameters yields the possibility of substantial insight into what factors 
may be responsible for angiogenesis, or its failure, within the structural unit. This is investigated leading to a novel modelling prediction of how one should select between one of two potentially optimal means of increasing the system's angiogenic response.

The outline of this paper is as follows: in Section 2 the model is developed, while in Section 3 numerical travelling wave solutions are presented. In Section 4, we investigate the model analytically using perturbation theory. Finally, in Section 5, the relevance, implications and future uses of the model and its associated results are discussed.

\section{The model}

The model will describe healing of a slit excisional wound which is much longer than it is wide, and much wider than it is deep, in those parts of the wound far from the corners. Thus one may consider a model with one spatial degree of freedom with reflection symmetry about a plane drawn parallel to the long edges of the wound and equidistant from both. As we are not explicitly considering the mechanisms of initial endothelial cell stimulation, we consider the dynamics only after a small (relative to the capillary tip carrying capacity) initial distribution of active sprout tips has formed at the wound edge. The averaged capillary tip and endothelial cell (i.e. blood capillary vessel building block) densities are respectively denoted by $n(x, t)$ and $b(x, t)$ at position $x$ and time $t$. We choose $x=0$ to denote the reflection plane of symmetry described above. Note that for simplicity, the variable $b$ represents both new and old capillaries.

\subsection{The capillary tip density equation}

For the tip concentration $n$, we use the standard form of the conservation equation

$$
\frac{\partial n}{\partial t}=-\frac{\partial J(n)}{\partial x}+f(n, b)
$$

where $J(n)$ is the tip flux and $f(n, b)$ describes the tip kinetics. We take the tip flux to be

$$
J(n)=-D_{1} \frac{\partial n}{\partial x}-D_{2} n \frac{\partial b}{\partial x} .
$$

This flux term captures the phenomenologically reasonable supposition that capillary tips migrate via a biased random walk with such bias, modelled by the $-D_{2} n \partial b / \partial x$ term, in the direction of decreasing blood vessel density.

We briefly note this term replaces the bias of capillary tips in previous models; for example, it replaces the chemotactic bias in [13] and the haptotactic bias in [10] (though the model in this article has no analogue of the haptokinesis considered [10]). One can compare the contribution to the capillary tip flux from chemotaxis in [13], to the term $-D_{2} n \partial b / \partial x$ as predicted by their model, and observe that they have the same qualitative features for a travelling wave solution, exhibiting peaks within the front of the travelling wave. See, for example, figures 2(a), 2(b) and (5) in [13]. Analogous comments apply on comparing the haptotatic flux and 


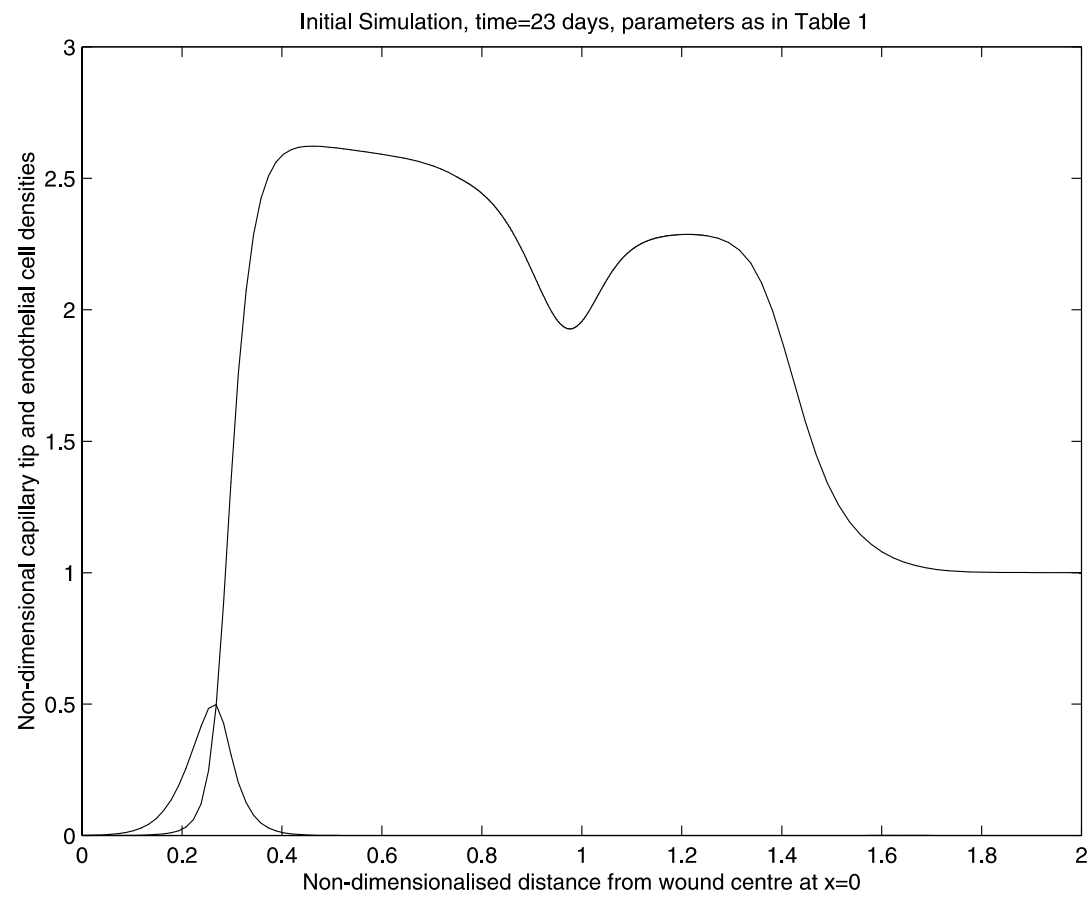

Fig. 2. An initial simulation, at 23 days, of the model with parameters given in Table 1 showing the development of a wave-like profile, possessing a 'structural unit'. To distinguish between the capillary tip density and the endothelial cell density in this figure, note that the capillary tip density is (essentially) zero for large $x$, whereas the blood vessel density is unity. This applies for all similar figures below

$-D_{2} n \partial b / \partial x$ in [10]. This, along with relative simplicity, provides the motivation for considering the $-D_{2} n \partial b / \partial x$ term as a model for bias in the capillary tip flux. We take the tip kinetics to be

$$
f(n, b)=\underbrace{\lambda_{2} n}_{\text {tip branching }}-\underbrace{\lambda_{3} n^{2}}_{\text {tip-tip anastomosis }}-\underbrace{\lambda_{4} n b}_{\text {tip-sprout anastomosis }} .
$$

The term $\lambda_{2} n$ describes the splitting of a tip into two separate branches; it is assumed that in a given length of time, a fixed proportion of existing sprouts will branch, while the term $-\lambda_{3} n^{2}$ describes the joining of two tips to create a complete circuit, and $-\lambda_{4} n b$ describes the joining of a tip to the side of a capillary, producing a complete circuit. Note that such terms for modelling branching anastomosis were first used by Edelstein in a model of fungal growth [20], and have since been applied on numerous occasions in modelling angiogenesis $[13,19]$.

\subsection{The endothelial cell density equation}

We assume that the capillary sprouts are not capable of independent movement, but instead that their movement is determined by the fact that they passively follow 
their leading tip. This yields proportionality between the flux of the capillary tips and the contribution to the endothelial cell flux arising from the invasion of the capillary tips into the wound bed. Thus we have that the flux of the endothelial cell density is given by

$$
-\lambda_{5}\left(D_{1} \frac{\partial n}{\partial x}+D_{2} n \frac{\partial b}{\partial x}\right)
$$

where $\lambda_{5}$ is the (average) number of endothelial cells in a capillary tip.

The kinetics of the endothelial cells are phenomologically modelled by

$$
g(n, b) \equiv \underbrace{\lambda_{6} v b\left(b_{0}-b\right)}_{\text {ordinary logistic remodelling }}+\underbrace{\lambda_{6} \chi n b\left(b_{1}-b\right)}_{\text {tip-modulated logistic growth }}+\underbrace{\lambda_{5}\left(\lambda_{3} n^{2}+\lambda_{4} n b\right)}_{\text {endothelial cells from the capillary tip compartment }} .
$$

This models three processes. The first, ordinary logistic remodelling, describes the proliferation of endothelial cells under standard conditions. This process has a carrying capacity of $b_{0}$, and takes place at a rate characterised by $v$. During angiogenesis a different contribution to the kinetics, occurring on a faster timescale, is observed in the immediate vicinity of a capillary tip [2, 4]. This is captured phenomenologically in the second term which possesses a characteristic rate proportional to the capillary tip density, $n$ and a carrying capacity of $b_{1}$. The fact this process proceeds at a faster rate entails that $n_{\max } \chi \gg v$, as discussed further in Appendix (A.2), where parameter estimation is detailed. The third process describes the contribution to the endothelial cell density kinetics arising from anastomosis (see equation (3)). Finally, note that the ordinary logistic modelling term cannot be simply ignored as one may readily anticipate regions of the spatial domain where $\chi n \ll v$.

\subsection{Initial and boundary conditions}

We suppose that initially there are neither capillaries nor tips in the wound space, and that the capillary density outside the wound is at the normal unwounded level, $b_{0}$. We assume an initial perturbation of capillary tip density which lies in a band of width $\varsigma$ at the wound edge and is small (compared to the characteristic scale of the carrying capacity for the capillary tip kinetics). The equations are to be solved on the domain $x \in[0, \infty)$, where $x=0$ is the wound centre and $x=x_{0}$ the wound edge. The initial conditions are

$$
\begin{gathered}
n(x, 0)= \begin{cases}0 & x \in\left[0, x_{0}\right), \\
n_{\text {init }} & x \in\left[x_{0}, x_{0}+\varsigma\right], \\
0 & x \in\left(x_{0}+\varsigma, \infty\right),\end{cases} \\
b(x, 0)= \begin{cases}0 & x \in\left[0, x_{0}\right), \\
b_{0} & x \in\left[x_{0}, \infty\right) ;\end{cases}
\end{gathered}
$$

though in numerical simulations we smooth the edges of these step-function-type conditions. 
For the boundary conditions, we have that far from the wound, at $x=\infty$, the vessel density remains that of the normal unwounded dermis and the tip density is zero. Also, as the wound is symmetric about its centre, we can apply the symmetric boundary condition at $x=0$, and hence

$$
\begin{gathered}
n(\infty, t)=0, \quad b(\infty, t)=b_{0}, \\
\frac{\partial n}{\partial x}(0, t)=\frac{\partial b}{\partial x}(0, t)=0 .
\end{gathered}
$$

\subsection{The nondimensionalised equations}

Details of the nondimensionalisation can be found in Appendix A.1. Omitting tildes used in this appendix for notational simplicity, the nondimensionalised model is

$$
\begin{aligned}
\frac{\partial n}{\partial t} & =\underbrace{\frac{\partial}{\partial x}\left[C_{1} \frac{\partial n}{\partial x}\right]}_{\text {diffusion }}+\underbrace{\frac{\partial}{\partial x}\left[C_{2} n \frac{\partial b}{\partial x}\right]}_{\text {movement away from capillaries }}+\underbrace{f(n, b),}_{\text {tip kinetics }} \\
\frac{\partial b}{\partial t} & =\underbrace{k_{5} \frac{\partial}{\partial x}\left[C_{1} \frac{\partial n}{\partial x}+C_{2} n \frac{\partial b}{\partial x}\right]}_{\text {capillaries follow tips }}+\underbrace{g(n, b)}_{\text {capillary kinetics }} ; \\
g(n, b) & =\chi n \beta b\left(1-\frac{b}{\beta}\right)+v b(1-b)+k_{2} n-k_{3} n^{2}-k_{3} n b, \\
& =\chi n \beta_{1} b\left(1-\frac{b}{\beta_{1}}\right)+v b(1-b)+k_{5} k_{4} n b
\end{aligned}
$$

where $\beta_{1} \stackrel{\text { def }}{=} \beta+k_{5} k_{4} / \chi$. The parameters $C_{1}, C_{2}, k_{2}, k_{3}, k_{4}, k_{5}, \chi, \nu, \beta, \varsigma$ and $n_{\text {init }}$ are non-negative constants resulting from the rescalings of $D_{1}, D_{2}, \lambda_{2}, \lambda_{3}$, $\lambda_{4}, \lambda_{5}, \chi, v, b_{1}, \varsigma$ and $n_{\text {init }}$. The initial and boundary conditions are

$$
\begin{gathered}
n(x, 0)= \begin{cases}0 & x \in[0,1), \\
n_{\text {init }} & x \in[1,1+\varsigma], \\
0 & x \in(1+\varsigma, \infty),\end{cases} \\
b(x, 0)= \begin{cases}0 & x \in[0,1), \\
1 & x \in[1, \infty),\end{cases} \\
n(\infty, t)=0, \quad b(\infty, t)=1, \\
\frac{\partial n}{\partial x}(0, t)=\frac{\partial b}{\partial x}(0, t)=0 .
\end{gathered}
$$




\subsection{Steady states of the model}

For future reference, we briefly consider the steady states of the model. Taking $k_{4}>k_{2}$, as motivated in Appendix (A.2), there are three non-negative steady-states given by

$$
(n, b)=(0,0),(0,1),\left(\frac{k_{2}}{k_{3}}=1,0\right) .
$$

The first of these corresponds to the wounded steady-state. The second is the unwounded steady-state and the third is a biologically irrelevant state consisting of capillary tips and no blood vessels. One can readily deduce that, on an infinite domain with Neumann conditions, and subject to spatially homogeneous perturbations that the steady-state $(n, b)=(0,1)$ is stable, whereas the steady-states $(n, b)=(0,0),\left(\frac{k_{2}}{k_{3}}=1,0\right)$ are both unstable, as one would intuitively expect.

\section{Initial simulations}

The numerical method used is discussed in Appendix B, while parameter estimation is discussed in detail in Appendix A.2, and is summarised in Table 1.

Table 1. Reference parameter set. Note that we fix $k_{3}=k_{2}$. This, together with all aspects of parameter estimation, is detailed in Appendix A.2.

\begin{tabular}{||c|c|c||}
\hline$C_{1}=3.5 \times 10^{-4}$ & $k_{2}=0.83$ & $\beta=9.29$ \\
$C_{2}=3.5 \times 10^{-4}$ & $k_{3}=0.83$ & $\chi=0.3$ \\
$k_{4}=0.85$ & $v=2.5 \times 10^{-3}$ & $n_{\text {init }}=0.25$ \\
$k_{5}=0.25$ & $\varsigma=0.25$ & $\beta_{1}=10.0$ \\
\hline
\end{tabular}

\subsection{Initial simulations and observations}

The simulation in Figure 2 shows the development of a wave-like profile progressing towards the centre of the wound. Note that the solution has the qualitative features empirically observed in cutaneous wound healing. It exhibits a 'structural unit', whereby a unit consisting of a peak in the capillary tips, closely followed by a peak in blood vessel density, move in concert towards the wound centre; it also exhibits elevated blood vessel density throughout the wounded region, as one would expect with the formation of granulation tissue in the wound.

Note that in Figure 2 there is a secondary peak in the endothelial cell density $b$ near $x=1$ which arose during the transitory period, before the development of the front of the wave profile. Its persistence is due to the fact that a travelling wave has not yet developed on the domain. Indeed, the secondary peak in the tail of the endothelial cell density, $b$, is not translated as time increases, as can be seen by comparing Figures 2 and 3.

One can understand why travelling waves do not develop given the assumption that $n \rightarrow 0$ much faster than $b \rightarrow 1$ for relatively large $x$, as the solution approaches the unwounded steady state $(n, b)=(0,1)$. Such an assumption is consistent with Figures 2 and 3. To presume otherwise would be biologically counter-intuitive, 


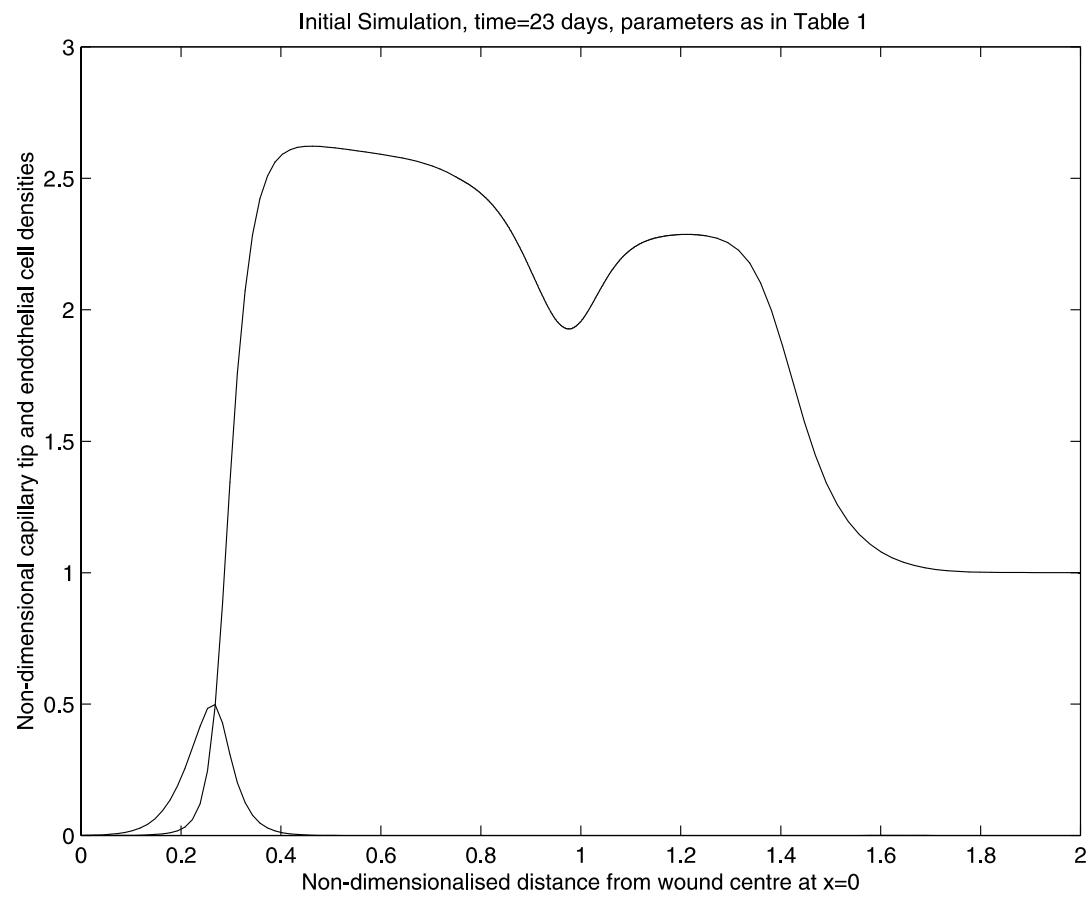

Fig. 3. An initial simulation, at 20 days, of the model with parameters given in Table 1 . Comparison with Figure 2 clearly shows that a travelling wave does not develop, in that the profile of the endothelial cell density (i.e. the profile which is essentially unity for large $x$ ) is not simply translated with increasing time

in that we anticipate there should be a significant density of capillary tips only at, or near, the edge of the invading structural unit, but a raised concentration of blood vessels in the forming granulation tissue at, and behind, the invading structural unit. With this assumption, we consider the endothelial cell density equation, transformed into travelling wave coordinates. Thus we have

$$
k_{5} C_{1} \hat{N}^{\prime \prime}+k_{5} C_{2}\left(\hat{N} \hat{B}^{\prime}\right)^{\prime}-c \hat{B}^{\prime}+g(\hat{N}, \hat{B})=0
$$

where $z=x+c t$, with $c>0$ the travelling wave speed, ' denotes $d / d z$ and $\hat{N}(z)=n(x, t), \hat{B}(z)=b(x, t)$. On linearising this equation about the unwounded steady state $(n, b)=(0,1)$, with the above assumption, $|b-1|=|\hat{B}-1| \ll n=\hat{N}$, one can readily show that $\hat{B}=1+E e^{-v z / c}$ where $E$ is a constant of integration. Thus, the (non-dimensionalised) characteristic lengthscale of the decay for the endothelial cell density tail on approaching the unwounded steady state $(n, b)=(0,1)$ for large $z$ is given by $c / v \sim 400 c$, while the non-dimensionalised wound domain occupies the unit interval. Consequently, providing the characteristic speed of the wave is sufficiently large (we find later $400 c \sim O(10)$ ), the tail of the travelling wave never forms on a biologically realistic domain size, as the travelling wave tail decays on a much larger spatial scale than the scale of a realistic domain. 
This can be confirmed by seeing if a travelling wave type solution forms if $v$ is increased (even if such an increase is not biologically realistic). The resulting $(n, b)$ profile has been plotted in Figure 4, and a much steeper $b$ tail is exhibited. This profile is further observed to be that of a travelling wave (to an excellent approximation), whereby it is simply translated with increasing time (not shown).

Even when travelling wave solutions are not seen in wound healing models, owing to the fact that the realistic domain size is too small to allow them to develop from the initial conditions, features of the travelling wave solutions can prove to be useful approximations for many features of the wave-like solutions of the full partial differential equations (see e.g. [11,12]). Thus, we will examine the travelling waves associated with this system, deriving estimates for the speed of the "structural unit', i.e. the concentration of capillary tips and blood vessels, and approximations for the peak densities of capillary tips and blood vessels. We will proceed to verify that such approximations hold for the solutions to the partial differential equations to a good approximation for numerous variations in the model parameters, which will greatly facilitate further investigation.

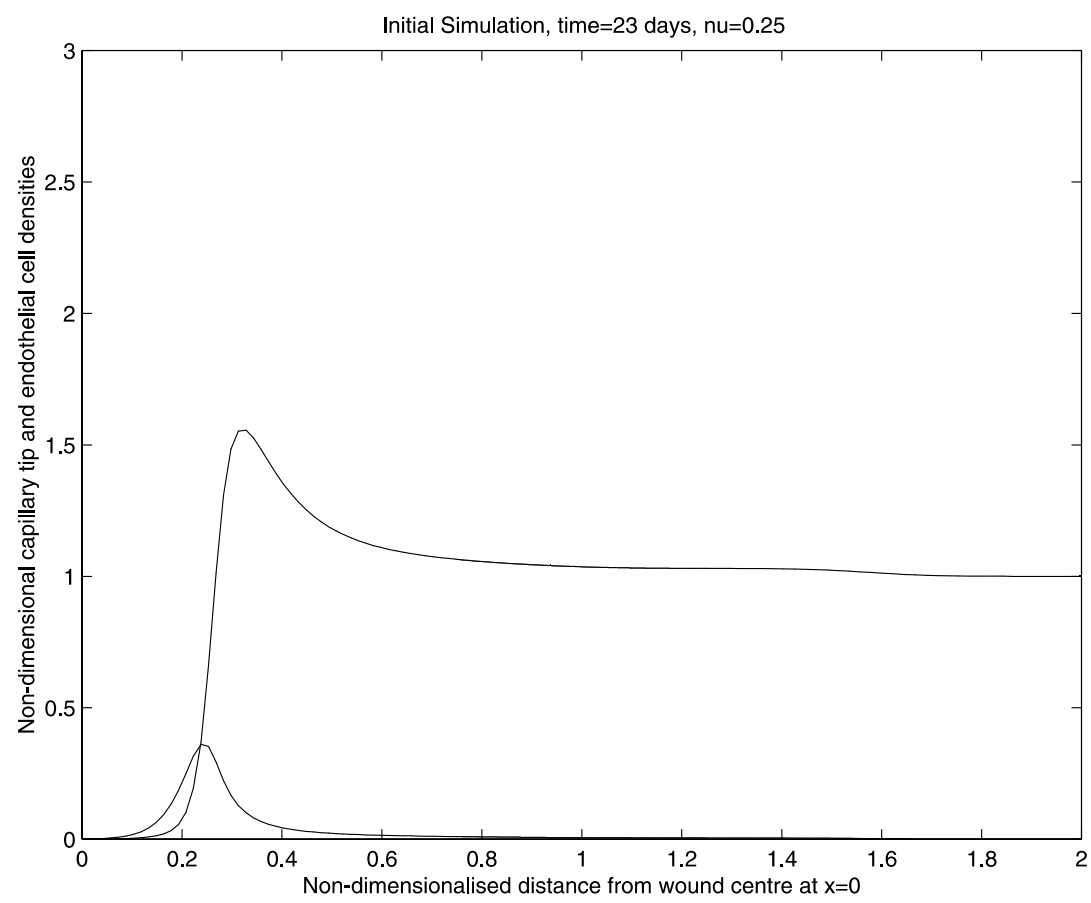

Fig. 4. Here the parameters are as given in Table 1, except that $v$ has been increased to 0.25 . Note that the endothelial cell density tail decays to its asymptotic value of unity. One may further observe from the numerics that the resulting $(n, b)$ profile evolves to a travelling wave (to an excellent approximation), whereby the above profile is simply translated (not shown) 


\section{Detailed travelling wave analysis}

\subsection{Travelling wave speed of the structural unit}

Consider the travelling wave coordinate $z=x+c t$, where $c>0$ is the wave speed, and the travelling wave variables $\hat{N}(z)=n(x, t), \hat{B}(z)=b(x, t)$. The model equations become

$$
\begin{gathered}
C_{1} \hat{N}^{\prime \prime}+C_{2}\left(\hat{N} \hat{B}^{\prime}\right)^{\prime}-c \hat{N}^{\prime}+f(\hat{N}, \hat{B})=0, \\
k_{5} C_{1} \hat{N}^{\prime \prime}+k_{5} C_{2}\left(\hat{N} \hat{B}^{\prime}\right)^{\prime}-c \hat{B}^{\prime}+g(\hat{N}, \hat{B})=0 ;
\end{gathered}
$$

where the prime denotes $d / d z$. Far behind the wave is unwounded dermis and far ahead of the wave is the unhealed wound, so the boundary conditions are $\hat{B}(\infty)=1$, $\hat{B}(-\infty)=0, \hat{N}(\infty)=0, \hat{N}(-\infty)=0$.

A standard investigation of the eigenvalues at the steady states $(\hat{N}, \hat{B})=(0,0)$ yields a minimum wave speed, given by

$$
c^{2} \geq 4 C_{1} k_{2} .
$$

Note that, on assuming a spatially infinite domain, the model's initial conditions are positive semi-definite for both $n$ and $b$, the initial conditions for $n$ are of compact support and finally that the initial conditions for $b$ satisfy $b=0$ for all $x<K$ for some finite $K$. Given such constraints on initial conditions, we hypothesise that the minimum wavespeed of the travelling wave yields a good estimate for the speed of propagation of the structural unit (i.e. the capillary tip peak and endothelial cell front) observed in Figures 3 and 4. Rigorous proofs of analogous hypotheses are well-known for scalar equations, but are typically lacking for systems of equations, especially those exhibiting non-monotonicity. However, our above hypothesis is completely consistent with all our numerical observations on the behaviour of this model. For example, one can compare Figures 2 and 3 to see that the wave profile has propagated a non-dimensionalised distance of approximately 0.1 (more accurately 0.105 ) in three time units (days), giving a non-dimensionalised speed of 0.035 , whereas $\sqrt{4 C_{1} k_{2}}=0.040$. The discrepancy between the observed speed and the minimum travelling wave speed is relatively small and arises due to the fact the wave front is still evolving to its attractor on the biological domain. We briefly note that this discrepancy has been observed to tend to zero at large times on a biologically unrealistic, extended, wound bed. Such observations are well-known and frequently made for the form of reaction diffusion systems commonly used in wound healing modelling $[11,12]$. Thus we have, as is standard, the observation that the propagation speed of the structural unit is accurately approximated by a single parameter grouping.

\subsection{Asymptotic investigation of the structural unit}

We proceed to study the travelling wave equations in more detail. Since the model has more than one small parameter, the variables are rescaled and new parameter groupings defined in order to express the equations in terms of only one explicit 
small parameter, $\epsilon$. The equations are subsequently investigated via an expansion in $\epsilon$, with the aim of extracting analytical information concerning the structural unit. In particular, we focus on the maxima of the endothelial cell density within the structural unit, as this represents a measure of the system's angiogenic ability. In the following we have three distinct asymptotic regions which are matched (or rather "patched") at two distinct points. We subsequently determine the approximation for the endothelial cell density maximum in terms of model parameters, and illustrate the correlation between our analytic predictions, and the numerical model.

A convenient rescaling of the non-dimensionalised PDE model is

$$
\tau=k_{2} t, \quad y=\sqrt{\frac{k_{2}}{C_{1}}} x, \quad \hat{n}=\frac{k_{3}}{k_{2}} n
$$

which gives (on dropping the hat for convenience)

$$
\begin{aligned}
\frac{\partial n}{\partial \tau}= & \frac{\partial^{2} n}{\partial y^{2}}+\frac{C_{2}}{C_{1}} \frac{\partial}{\partial y}\left[n \frac{\partial b}{\partial y}\right]+\left(n-n^{2}\right)-\frac{k_{4}}{k_{2}} n b, \\
\frac{\partial b}{\partial \tau}= & k_{5}\left(\frac{\partial^{2} n}{\partial y^{2}}+\frac{C_{2}}{C_{1}} \frac{\partial}{\partial y}\left[n \frac{\partial b}{\partial y}\right]\right)+\left(\frac{\chi \beta_{1}}{k_{3}} n+\frac{v}{k_{2}}\right) b-\left(\frac{\chi}{k_{3}} n+\frac{v}{k_{2}}\right) b^{2} \\
& +\frac{k_{5} k_{2}}{k_{3}} n^{2} .
\end{aligned}
$$

Some of the coefficients of the above terms are of order 1 , so we define $O(1)$ parameters as follows:

$$
\rho_{1}=\frac{C_{2}}{C_{1}} \sim O(1), \quad \rho_{2}=\frac{k_{4}}{k_{2}} \sim O(1), \quad \rho_{3}=\frac{\chi}{k_{3}} \sim O(1) .
$$

We now transform to travelling wave coordinate

$$
\bar{z}=y+\bar{c} \tau,
$$

with the variables

$$
\bar{N}(\bar{z})=n(y, \tau), \quad \bar{B}(\bar{z})=b(y, \tau) .
$$

The equations become

$$
\begin{gathered}
\frac{d^{2} \bar{N}}{d \bar{z}^{2}}+\rho_{1} \frac{d}{d \bar{z}}\left[\bar{N} \frac{d \bar{B}}{d \bar{z}}\right]-\bar{c} \frac{d \bar{N}}{d \bar{z}}+\left(\bar{N}-\bar{N}^{2}\right)-\rho_{2} \bar{N} \bar{B}=0, \\
\left(\frac{d^{2} \bar{N}}{d \bar{z}^{2}}+\rho_{1} \frac{d}{d \bar{z}}\left[\bar{N} \frac{d \bar{B}}{d \bar{z}}\right]\right)+\rho_{1} \bar{N} \frac{d \bar{B}}{d \bar{z}}-\bar{c} \frac{d \bar{B}}{d \bar{z}}+\left(\bar{B} \beta_{1} \rho_{3} \bar{N}+\frac{v}{k_{2}}\right) \bar{B} \\
-\left(\rho_{3} \bar{N}+\frac{v}{k_{2}}\right) \bar{B}^{2}+\frac{k_{5} k_{2}}{k_{3}} \bar{N}^{2}=0,
\end{gathered}
$$


with the travelling wave boundary conditions

$$
\bar{B}(\infty)=1, \bar{B}(-\infty)=0, \bar{N}(\infty)=0, \bar{N}(-\infty)=0 .
$$

Note that the original travelling wave coordinate was

$$
z=x+c t=\sqrt{\frac{C_{1}}{k_{2}}} y+\frac{c}{k_{2}} \tau=\sqrt{\frac{C_{1}}{k_{2}}}\left(y+\frac{c}{\sqrt{C_{1} k_{2}}} \tau\right) .
$$

So if we set $\bar{c}=\frac{c}{\sqrt{C_{1} k_{2}}}$ we have $z=\sqrt{\frac{C_{1}}{k_{2}}} \bar{z}$. The theoretical minimum of $c$ is $\sqrt{4 C_{1} k_{2}}$, hence

$$
\bar{c} \geq 2
$$

so we can use $\bar{c}$ to define a small parameter

$$
\epsilon=\frac{1}{\bar{c}^{2}} \leq \frac{1}{4}
$$

Given the above observations that the front of the structural unit attains the minimum travelling wave speed, we hypothesise that the trajectory in phase space which actually corresponds to the PDE model attractor is the one of minimum travelling wavespeed for non-negative solutions. Thus below we take

$$
\bar{c}=2, \quad \epsilon=\frac{1}{4} .
$$

For convenience, we define the $O(1)$ parameter, $\rho_{4}$ by

$$
\rho_{4}=\frac{v}{k_{2}} \epsilon^{-4}
$$

\subsection{Inner and outer solutions}

Consider a general rescaling based on $\epsilon$ :

$$
\xi_{\sigma}=\epsilon^{\sigma+\frac{1}{2}} \bar{z}, \quad \bar{N}(\bar{z})=N(\xi), \quad \bar{B}(\bar{z})=B(\xi),
$$

where $\sigma$ will be chosen to give the appropriate scaling. The only value of $\sigma$ which will allow us to match algebraic terms with a derivative, as required for nontrivial behaviour, is $\sigma=0$. The travelling wave equations then become

$$
\begin{aligned}
\epsilon \frac{d^{2} N}{d \xi_{0}^{2}}+\rho_{1} \epsilon \frac{d}{d \xi_{0}}\left[N \frac{d B}{d \xi_{0}}\right]-\frac{d N}{d \xi_{0}}+\left(N-N^{2}\right)-\rho_{2} N B=0 \\
k_{5} \epsilon\left(\frac{d^{2} N}{d \xi_{0}^{2}}+\rho_{1} \frac{d}{d \xi_{0}}\left[N \frac{d B}{d \xi_{0}}\right]\right)-\frac{d B}{d \xi_{0}}+\left(\rho_{3} \beta_{1} N+\rho_{4} \epsilon^{4}\right) B-\left(\rho_{3} N+\rho_{4} \epsilon^{4}\right) B^{2} \\
+\frac{k_{5} k_{2}}{k_{3}} N^{2}=0 .
\end{aligned}
$$




\subsubsection{Inner solution}

The numerical simulations of the previous sections show that while there exists an 'inner' region with $N, B \gg \epsilon^{4}, N$ and $B$ are both small in the wavefront, and $N$ is small in the waveback; see Figure 5. To capture the inner solution, we use a standard expansion

$$
\begin{aligned}
& N=N_{0}+\epsilon N_{1}+\epsilon^{2} N_{2}+\cdots, \\
& B=B_{0}+\epsilon B_{1}+\epsilon^{2} B_{2}+\cdots,
\end{aligned}
$$

with the implicit assumption that $B, N \sim O(1)$ at leading order. Ignoring terms of $O(\epsilon)$, equations (24) and (25) yield

$$
\begin{gathered}
\frac{d N_{0}}{d \xi_{0}}=N_{0}\left(1-N_{0}-\rho_{2} B_{0}\right), \\
\frac{d B_{0}}{d \xi_{0}}=N_{0} \rho_{3} \beta_{1}\left[\left(1-\frac{B_{0}}{\beta_{1}}\right) B_{0}+\frac{k_{5} k_{2}}{k_{3} \rho_{3} \beta_{1}} N_{0}\right]
\end{gathered}
$$

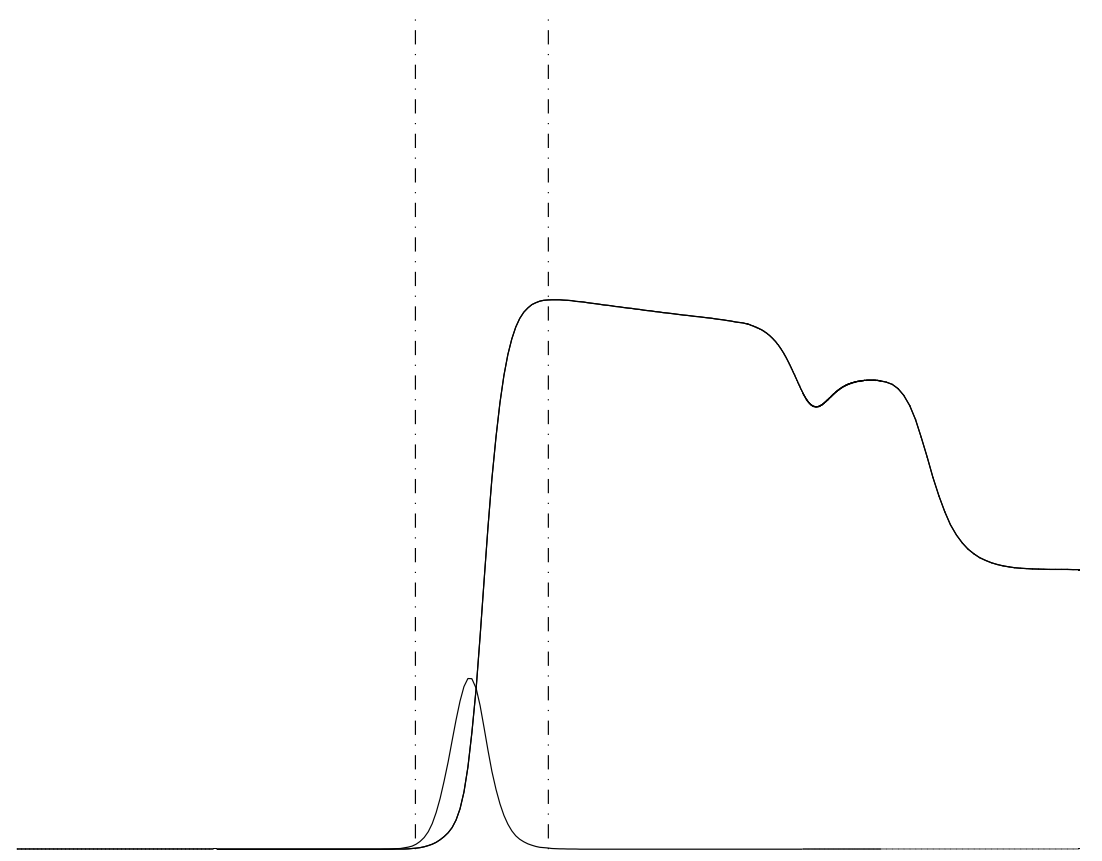

Fig. 5. Schematic indicating the three asymptotic regions of the travelling wave; the higher peak corresponds to the endothelial cell density, the lower peak to capillary tip density. Between the two dashed lines lies the inner region. To the left of inner region is the outer wavefront region, whereas to the right of the inner region is the outer waveback region. The left-hand dashed line indicates the approximate position of the first matching point (on the spatial domain). This is denoted matching point 1 . Similarly the right hand dashed line indicates the approximate position of the second matching point, which is denoted matching point 2 
However, as motivated in Appendix A. $2, k_{5} /\left(\rho_{3} \beta_{1}\right) \ll 1$ may be treated as asymptotically small. Thus, we have, at leading order, the approximation

$$
\frac{d B_{0}}{d \xi_{0}}=N_{0} \rho_{3} \beta_{1}\left(1-\frac{B_{0}}{\beta_{1}}\right) B_{0} .
$$

Dividing equation (26) by equation (28), and integrating, we have

$$
N_{0}=\left(\frac{\beta_{1}-B_{0}}{B_{0}}\right)^{\frac{1}{\rho_{3} \beta_{1}}}\left[D+H\left(d, B_{0}\right)\right]
$$

where

$$
H(a, b)=\int_{a}^{b} \frac{1-\rho_{2} B^{\prime}}{\rho_{3} \beta_{1} B^{\prime}\left(1-\frac{B^{\prime}}{\beta_{1}}\right)}\left(\frac{B^{\prime}}{\beta_{1}-B^{\prime}}\right)^{\frac{1}{\rho_{3} \beta_{1}}} d B^{\prime}
$$

and $D, d$ are constants related by whatever choice is made for the lower limit, $d$, of the integration.

\subsubsection{Outer waveback solution}

We now consider the outer solution in the waveback. Note that essentially when we ignored terms of $O(\epsilon)$ in the inner solution, we were assuming that the $\rho_{4} \epsilon^{4}$ terms in (25) were dominated by the $\rho_{3} \beta_{1} N$ and $\rho_{3} N$ terms. This will not be the case in the outer waveback region, as we require $N \rightarrow 0$ as $\xi_{0} \rightarrow \pm \infty$. Thus we assume that $N$ is of order not more than $\epsilon^{4}$, and so try a perturbation expansion of the form

$$
\begin{aligned}
& N=\epsilon^{4} N_{0}+\epsilon^{5} N_{1}+\cdots, \\
& B=B_{0}+\epsilon B_{1}+\epsilon^{2} B_{2}+\cdots .
\end{aligned}
$$

Substituting into equation (24) and (25) gives, at leading order,

$$
\begin{gathered}
\frac{d N_{0}}{d \xi_{0}}=N_{0}\left[1-\rho_{2} B_{0}\right], \\
\frac{d B_{0}}{d \xi_{0}}=\rho_{4} \epsilon^{4} B_{0}\left(1-B_{0}\right) .
\end{gathered}
$$

The term of order $\epsilon^{4}$ is retained in the latter equation, as required for consistency with the boundary conditions. (No appropriate scaling will remove the $\epsilon^{4}$, and without the term's retention one would quickly come across inconsistencies (see below)). Dividing and integrating,

$$
N_{0}=K B_{0}^{\frac{1}{\rho_{4} \epsilon^{4}}}\left|1-B_{0}\right|^{\frac{\rho_{2}-1}{\rho_{4} \epsilon^{4}}}
$$

where $K$ is a constant. 


\subsubsection{Outer wavefront solution}

Analogously to the waveback, we take it that $N$ is not of order more than than $\epsilon^{4}$ in the wavefront, as the boundary of the wavefront and inner region is similarly determined according to whether the $\rho_{4} \epsilon^{4}$ terms in (25) dominate the $\rho_{3} \beta_{1} N$ and $\rho_{3} N$ terms. We also assume in the wavefront that $B$ is small. Thus we consider an expansion of the form

$$
\begin{aligned}
& N=\epsilon^{4} N_{0}+\epsilon^{5} N_{1}+\cdots, \\
& B=\epsilon^{\mu}\left(B_{0}+\epsilon B_{1}+\epsilon^{2} B_{2}+\cdots\right), \quad \mu>0 .
\end{aligned}
$$

Use of the expansion (34) in equation (25) only gives a nontrivial leading order perturbative approximation for $\mu=5$, whereupon

$$
B_{0}^{\prime}=k_{5} N_{0}^{\prime \prime}\left(1+O\left(\epsilon^{4} B_{0}, \epsilon^{4} B_{0}^{\prime}, \epsilon^{4} B_{0}^{\prime \prime}\right)\right)
$$

Note that we do not expect any boundary layers in the wavefront, i.e. we do not expect to have to rescale $\xi_{0}$ due to large gradients in $N_{0}$ or $B_{0}$, and hence one does not have to apply matched asymptotic techniques to correctly incorporate higher order derivatives in a perturbative expansion of the wavefront equations. Thus the higher order terms in (35) are neglected, and one can deduce $B_{0}=k_{5} N_{0}^{\prime}\left(1+O\left(\epsilon^{4}\right)\right)$, with the constant of integration determined by boundary conditions as $\xi_{0} \rightarrow-\infty$. Substituting the expansion (33) into equation (24) yields $N_{0}^{\prime}=N_{0}(1+O(\epsilon))$. Hence in the waveback at leading order we have

$$
B_{0}=k_{5} N_{0}(1+O(\epsilon)) .
$$

\subsection{Solution matching}

For notational simplicity below, we denote the leading order approximation to $N$ and $B$ by the same symbols.

We will match when the $\rho_{4} \epsilon^{4}$ terms in equation (25) are the same size as the $\rho_{3} \beta_{1} N$ and $\rho_{3} N$ terms. So at each matching point, $\left|\rho_{3} \beta_{1} N B-\rho_{3} N B^{2}\right|=\mid \rho_{4} \epsilon^{4} B-$ $\rho_{4} \epsilon^{4} B^{2} \mid$, i.e.

$$
\left|\rho_{3} N\left(\beta_{1}-B\right)\right|=\left|\rho_{4} \epsilon^{4}(1-B)\right| .
$$

We need to match at two points, as described in Figure (5). Let $N_{(1)}, B_{(1)}$ be the values of $N$ and $B$ at the matching point between the inner region and the outer wavefront region, i.e. matching point 1 of Figure (5). Similarly, let $N_{(2)}, B_{(2)}$ denote the values of $N$ and $B$ at the matching point between the inner region and the outer waveback, i.e. matching point 2 of Figure (5).

At the first matching point, $B_{(1)}<1$ and thus (36), (37) give

$$
\begin{aligned}
B_{(1)} & =k_{5} \epsilon N_{(1)}(1+O(\epsilon)) \\
\rho_{3} N_{(1)}\left(\beta_{1}-B_{(1)}\right) & =\rho_{4} \epsilon^{4}\left(1-B_{(1)}\right) .
\end{aligned}
$$


The appearance of the factor of $\epsilon$ in relation (38) arises due to the different scalings of $B_{0}$ and $N_{0}$ in the wavefront. Taking $d=B_{(1)}$ in equation (29) gives

$$
N_{(1)}=D\left(\frac{\beta_{1}-B_{(1)}}{B_{(1)}}\right)^{\frac{1}{\rho_{3} \beta_{1}}} .
$$

Now consider the second matching point. Equation (28) shows that the only turning point of $B=B_{0}$ in the inner region for $N_{0} \neq 0$ is $B=B_{0}=\beta_{1}$. However, all numerical simulations suggest that $b_{\max }<\beta_{1}$. Similarly, equation (31) for $\frac{d B_{0}}{d \xi}$ in the outer waveback region shows that the only turning point of $B=B_{0}$ in the waveback for $N_{0} \neq 0$ is $B=B_{0}=1$; however, all numerical simulations suggest that $b_{\max }>1$. Hence as we approach the matching point from below, $B_{\text {inner }}$ increases towards $\beta_{1}$, and as we increase $\xi_{0}$ to pass beyond the matching point, $B_{\text {waveback }}$ decreases towards 1 . So $B_{(2)}$ is actually the maximum value of $B$ in our matched solution. Thus if we can find $B_{(2)}$ in terms of the parameters, we should have a good approximation to the value of $b_{\max }$. Thus, we explicitly anticipate that $B=B_{0}$ is not constant in the outer waveback, and thus one must maintain the $O\left(\epsilon^{4}\right)$ term in equation (31) for the outer waveback.

As we expect $1<B_{(2)}<\beta_{1}$ equations (32) and (37) give

$$
\begin{array}{r}
N_{(2)}=\epsilon^{4} K B_{(2)}^{\frac{1}{\rho_{4} \epsilon^{4}}}\left(B_{(2)}-1\right)^{\frac{\rho_{2}-1}{\rho_{4} \epsilon^{4}}}, \\
\rho_{3} N_{(2)}\left(\beta_{1}-B_{(2)}\right)=\rho_{4} \epsilon^{4}\left(B_{(2)}-1\right),
\end{array}
$$

while (29) implies

$$
N_{(2)}=\left(\frac{\beta_{1}-B_{(2)}}{B_{(2)}}\right)^{\frac{1}{\rho_{3} \beta_{1}}}\left[D+H\left(B_{(1)}, B_{(2)}\right)\right] .
$$

Note that we have six unknowns $\left(N_{(1)}, N_{(2)}, B_{(1)}, B_{(2)}, K\right.$ and $\left.D\right)$ and six equations. Thus, one can in principle solve to determine $N=N(B)$, and integrate to find $N\left(\xi_{0}\right), B\left(\xi_{0}\right)$. However, in practice, even analytically solving for $\left(N_{(1)}, N_{(2)}\right.$, $B_{(1)}, B_{(2)}, K$ and $D$ ) is too cumbersome to perform. One can make a number of important observations by considering the expressions for $\left(B_{(1)}, N_{(1)}, D\right)$, without solving for $\left(B_{(2)}, N_{(2)}, K\right)$. For future reference, we note that equations (39) and (40) can be readily solved to give

$$
\begin{gathered}
B_{(1)}=\frac{k_{5} \rho_{4}}{\rho_{3} \beta_{1}} \epsilon^{5}(1+O(\epsilon)), \quad N_{(1)}=\frac{\rho_{4}}{\rho_{3} \beta_{1}} \epsilon^{4}\left(1+O\left(\epsilon^{5}\right)\right), \\
D=\frac{\rho_{4}}{\rho_{3} \beta_{1}} \epsilon^{4}\left(\frac{k_{5} \rho_{4}}{\rho_{3} \beta_{1}^{2}} \epsilon^{5}\right)^{\frac{1}{\rho_{3} \beta_{1}}}\left(1+O\left(\frac{\epsilon}{\rho_{3} \beta_{1}}\right)\right) .
\end{gathered}
$$




\subsection{Capillary tip density maximum}

\subsubsection{Analytical approach}

In this section we use the above asymptotic framework to determine the maximum value of the capillary tip density, and we will proceed to verify this numerically for a number of parameter variations.

Since the inner equations (26), (28) are valid in the region where the capillary tip density is largest, and as $N=N_{0}, B=B_{0}$ in the inner region, the maximum value attained by $N$ occurs when $N=1-\rho_{2} B$. Hence

$$
N^{\max }=1-\rho_{2} B\left(N^{\max }\right) .
$$

We now seek $B\left(N^{\max }\right) ; N^{\max }$ itself can then easily be determined from (44). For simplicity of notation, we define

$$
B_{*}=B\left(N^{\max }\right)
$$

From equation (29), we have an expression for $N(B)=N_{0}\left(B_{0}\right)$, and hence

$$
\begin{aligned}
1-\rho_{2} B_{*} & =\left(\frac{\beta_{1}-B_{*}}{B_{*}}\right)^{\frac{1}{\rho_{3} \beta_{1}}}\left[D+H\left(B_{(1)}, B_{*}\right)\right] \\
& =\left(\frac{\beta_{1}-B_{*}}{B_{*}}\right)^{\frac{1}{\rho_{3} \beta_{1}}}\left[O\left(\epsilon^{4}\right)+H\left(B_{(1)}, B_{*}\right)\right] .
\end{aligned}
$$

One can simplify (30) to obtain

$$
\begin{aligned}
H(a, b)= & \left(1-\rho_{2} \beta_{1}\right)\left[\left(\frac{b}{\beta_{1}-b}\right)^{\frac{1}{\rho_{3} \beta_{1}}}-\left(\frac{a}{\beta_{1}-a}\right)^{\frac{1}{\rho_{3} \beta_{1}}}\right] \\
& +\rho_{2} \beta_{1} \int_{\left(\frac{a}{\beta_{1}-a}\right)^{\frac{1}{\rho_{3} \beta_{1}}}}^{\left(\frac{b}{\beta_{1}-b}\right)^{\frac{1}{\rho_{3} \beta_{1}}}} \frac{d s}{1+s^{\rho_{3} \beta_{1}}} .
\end{aligned}
$$

With the definitions

$$
\begin{aligned}
& \psi_{*}=\left(B_{*} /\left(\beta_{1}-B_{*}\right)\right)^{1 /\left(\rho_{3} \beta_{1}\right)} \\
& \psi_{1}=\left(B_{(1)} /\left(\beta_{1}-B_{(1)}\right)\right)^{1 /\left(\rho_{3} \beta_{1}\right)}=\left(\frac{k_{5} \rho_{4}}{\rho_{3} \beta_{1}} \epsilon^{5}\right)^{\frac{1}{\rho_{3} \beta_{1}}}\left(1+O\left(\frac{\epsilon}{\rho_{3} \beta_{1}}\right)\right)
\end{aligned}
$$

substituting (46) into (45), neglecting orders of $\epsilon^{4}$ yields,

$$
\begin{aligned}
\int_{0}^{\psi_{*}} \frac{d s}{1+s^{\rho_{3} \beta_{1}}} & =\frac{\psi_{*}}{1+\psi_{*}^{\rho_{3} \beta_{1}}}-\frac{\rho_{2} \beta_{1}-1}{\rho_{2} \beta_{1}} \psi_{1}+\int_{0}^{\psi_{1}} \frac{d s}{1+s^{\rho_{3} \beta_{1}}} \\
& =\frac{\psi_{*}}{1+\psi_{*}^{\rho_{3} \beta_{1}}}+\frac{1}{\rho_{2} \beta_{1}} \psi_{1}+O\left(\frac{\psi_{1} \psi_{1}^{\rho_{3} \beta_{1}}}{\beta_{1}} \sim \frac{\epsilon^{5}}{\beta_{1}^{2}}\right) .
\end{aligned}
$$


We now consider the anzatz $\psi_{*}^{\rho_{3} \beta_{1}} \ll 1$. Neglecting terms at order $\psi_{*}^{2 \rho_{3} \beta_{1}}$, we readily find

$$
\psi_{*}^{\rho_{3} \beta_{1}}=\left(\frac{\rho_{3} \beta_{1}+1}{\rho_{2} \rho_{3} \beta_{1}^{2}} \psi_{1}\right)^{\frac{\rho_{3} \beta_{1}}{\rho_{3} \beta_{1}+1}}
$$

Recall that the anzatz is valid providing

$$
\begin{aligned}
\psi_{*}^{\rho_{3} \beta_{1}}= & \left(\frac{\rho_{3} \beta_{1}+1}{\rho_{2} \rho_{3} \beta_{1}^{2}} \psi_{1}\right)^{\frac{\rho_{3} \beta_{1}}{\rho_{3} \beta_{1}+1}}=\left(\frac{\rho_{3} \beta_{1}+1}{\rho_{2} \rho_{3} \beta_{1}^{2}}\left(\frac{k_{5} \rho_{4}}{\rho_{3} \beta_{1}} \epsilon^{5}\right)^{\frac{1}{\rho_{3} \beta_{1}}}\right)^{\frac{\rho_{3} \beta_{1}}{\rho_{3} \beta_{1}+1}} \\
& \times\left(1+O\left(\frac{\epsilon}{1+\rho_{3} \beta_{1}}\right)\right) \ll 1
\end{aligned}
$$

which typically holds for biologically reasonable parameter values. With the parameters in Table 1, the left-hand side of (49) is approximately 0.02, and does not exceed 0.05 , on varying $\beta_{1}$, within the interval $[3,16]$. Thus, we have at leading order in $\epsilon$,

$$
n_{\text {max }}=\frac{k_{2}}{k_{3}}\left(1-\rho_{2} B_{*}\right)=\frac{k_{2}}{k_{3}}\left(1-\rho_{2} \beta_{1} \psi_{*}^{\rho_{3} \beta_{1}}\left(1+O\left(\psi_{*}^{\rho_{3} \beta_{1}}\right)\right)\right)
$$

where $\psi_{*}^{\rho_{3} \beta_{1}}$ is given in equation (49) (and the factor of $k_{2} / k_{3}$ arises from the rescaling (20)).

\subsubsection{Numerical verification}

In Figure 6 we compare the approximation (50) to the numerical value of $N_{\max }$ keeping all parameters are fixed as given in Table 1, except for $\beta$ which is varied within the extent of its biologically plausible range $[3,15]$, which results in a variation of $\beta_{1}$ over the range $[3.7,15.7]$. In Figure 7 we compare the approximation (50) to the numerical value of $N_{\max }$ keeping all parameters fixed as given in Table $1 \mathrm{ex}-$ cept, this time, for variations of $k_{5}$. The motivation for varying $\beta$ and $k_{5}$ is due to the fact these parameters are difficult to estimate empirically. One can readily observe that both Figures 6 and 7 show the approximation (50) yields the same qualitative trends as the numerical results for their respective parameter variations. The errors involved are relatively large for an $O(\epsilon)$ approximation; this is speculated to be due to the fact that the maximum of $N$ is particularly sensitive to the behaviour of the system close to the inner-outer wavefront interface, where the approximations implicit in the patching procedure are relatively inaccurate. One could improve these approximations by performing a matching, rather than a patching, procedure but this is not pursued for reasons of simplicity and as we are more interested in the endothelial cell density maximum, which is less sensitive to inaccuracies in the approximations at the interfaces inherent in the patching procedure. 


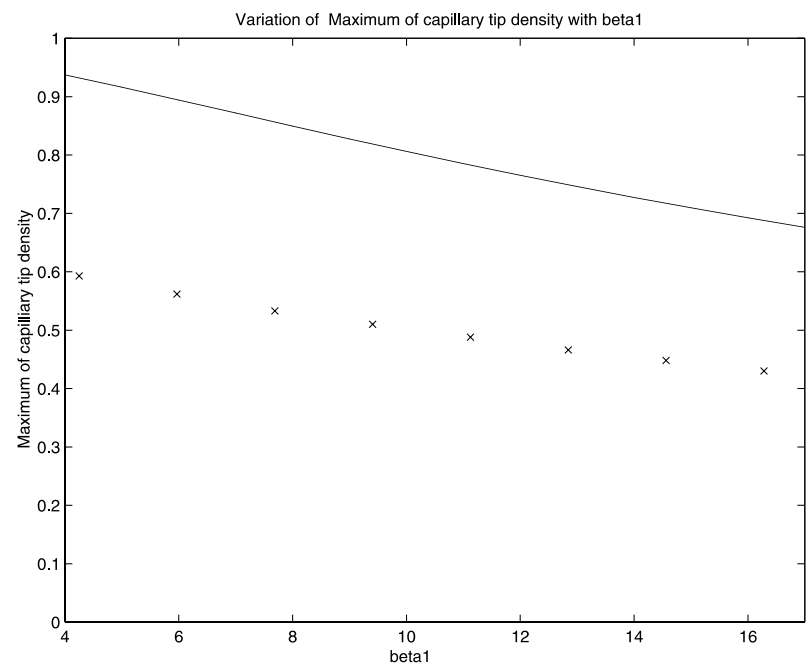

Fig. 6. Comparison of numerical maximum of $N$ (individually plotted) with approximation given in equation (50) (plotted as a solid line). All parameters are fixed as in Table 1, except $\beta_{1}$, which is varied in the interval $[4,16]$

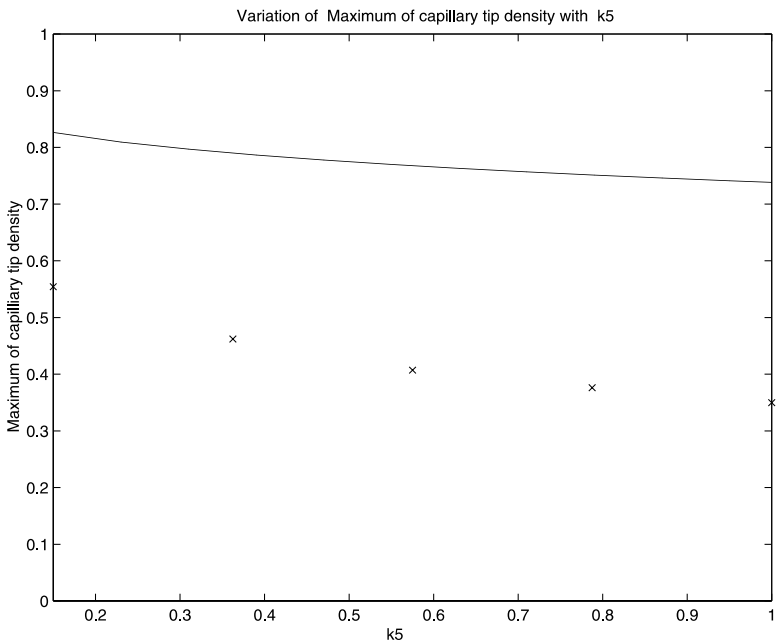

Fig. 7. Comparison of numerical maximum of $N$ (individually plotted) with approximation given in equation (50) (plotted as a solid line). All parameters are fixed as in Table 1, except $k_{5}$, which is varied in the interval $[0.1,1]$

\subsection{Endothelial cell density maximum}

In this section we investigate analytically the endothelial cell density maximum, which is a measure of the angiogenic ability of the system and its ability to supply nutrients to the wound healing structural unit. We will find, for example, that 
this maximum depends, at leading orders, on only a few parameter groupings. The implications of this, in the context of how one may attempt to induce a greater angiogenic response, will be investigated in detail in section (5.3).

\subsubsection{Perturbation theory}

We seek an approximation to $B_{(2)}$. Using (41) to eliminate $N_{(2)}$ from equation (42) gives, on rearrangement

$$
B_{(2)}=\frac{\rho_{4} \epsilon^{4}+\beta_{1}\left[\psi_{2}^{-1} \rho_{3}\left(D+H\left(B_{(1)}, B_{(2)}\right)\right)\right]}{\rho_{4} \epsilon^{4}+\left[\psi_{2}^{-1} \rho_{3}\left(D+H\left(B_{(1)}, B_{(2)}\right)\right)\right]}
$$

where

$$
\psi_{2}=\left(\frac{B_{(2)}}{\beta_{1}-B_{(2)}}\right)^{\frac{1}{\rho_{3} \beta_{1}}}
$$

Thus one has either that

$$
B_{(2)}=\beta_{1}-O\left(\epsilon^{4}\right)
$$

or, noting $D \sim O\left(\epsilon^{4}\right)$, that

$$
H\left(B_{(1)}, B_{(2)}\right) \leq O\left(\epsilon^{4}\right) \ll 1 .
$$

All observations from our numerical simulations indicate that $B_{(2)} \neq \beta_{1}-O\left(\epsilon^{4}\right)$, and thus we consider the latter. Neglecting orders of $\epsilon^{4}$, and using (46) we have

$$
\begin{aligned}
\left(1-\rho_{2} \beta_{1}\right) \psi_{2}+\rho_{2} \beta_{1} \int_{0}^{\psi_{2}} \frac{d x}{1+x^{\rho_{3} \beta_{1}}} & =\left(1-\rho_{2} \beta_{1}\right) \psi_{1}+\rho_{2} \beta_{1} \int_{0}^{\psi_{1}} \frac{d x}{1+x^{\rho_{3} \beta_{1}}} \\
& =\psi_{1}+O\left(\frac{\psi_{1} \psi_{1}^{\rho_{3} \beta_{1}}}{\beta_{1}} \sim \frac{\epsilon^{5}}{\beta_{1}^{2}}\right)
\end{aligned}
$$

As usual, we neglect terms of order $\epsilon^{4}$. The above equation for $\psi_{2}$ has two solutions; the smaller one gives $\psi_{2} \sim 0, B_{\max } \ll 1$, which is inappropriate and hence we seek the larger solution. In general, one should seek the solution to (51) to obtain the asymptotic estimate for $\psi_{2}$, and hence $B_{\max }$. However, noting $\psi_{1} \ll 1$ one may consider an expansion in terms of $\psi_{1}$, which will be valid in quite general circumstances, as investigated below. Defining the function

$$
F(p)=\left(1-\rho_{2} \beta_{1}\right) p+\rho_{2} \beta_{1} \int_{0}^{p} \frac{d x}{1+x^{\rho_{3} \beta_{1}}}
$$

we have $F\left(\psi_{2}\right)=\psi_{1}$ (neglecting the $O\left(\epsilon^{5} / \beta_{1}^{2}\right)$ term). Treating $\psi_{1}$ as asymptotically small, and writing $\psi_{2}$ as power series in $\psi_{1}$ gives, via standard perturbation techniques, that

$$
\psi_{2}=\psi_{2}^{0}+\frac{\psi_{1}}{F^{\prime}\left(\psi_{2}^{0}\right)}-\frac{F^{\prime \prime}\left(\psi_{2}^{0}\right) \psi_{1}^{2}}{2 F^{\prime 2}\left(\psi_{2}^{0}\right)}+\ldots
$$


where

$$
\frac{1}{\psi_{2}^{0}} \int_{0}^{\psi_{2}^{0}} \frac{d x}{1+x^{\rho_{3} \beta_{1}}}=\frac{\rho_{2} \beta_{1}-1}{\rho_{2} \beta_{1}} .
$$

This gives, at leading order in $\psi_{1}$ and $\epsilon$,

$$
\begin{gathered}
\psi_{2}=\psi_{2}^{0} \\
B_{\max }=B_{(2)}^{0}+O\left(\epsilon, \psi_{1}\right), \quad B_{(2)}^{0}=\frac{\beta_{1}\left(\psi_{2}^{0}\right)^{\rho_{3} \beta_{1}}}{1+\left(\psi_{2}^{0}\right)^{\rho_{3} \beta_{1}}} .
\end{gathered}
$$

This constitutes a particularly useful perturbative approximation for $B_{\max }$ which, as we will investigate below, is valid for biologically relevant parameters whenever $\beta_{1}$ is sufficiently small. For example, one requires $\beta_{1}<9$ when all other parameters are as given in Table 1).

\subsubsection{Correction terms and validity of the approximation $B_{\max }=B_{(2)}^{0}$.}

Before numerically investigating the above approximations for $B_{\max }$ we consider the validity of taking $\psi_{1}$ as asymptotically small in equation (54) by investigating the corrective terms in (52).

We consider the first correction term initially. As shown in Appendix (C), via the inequalities (70) and (71), one has

$$
\left|\frac{\psi_{1}}{F^{\prime}\left(\psi_{2}^{0}\right)}\right|<A \psi_{1} \leq(9 / 5) \psi_{1} \ll 1
$$

where $A \stackrel{\text { def }}{=} 1$ for $\beta_{1} \geq 5$ and $A \stackrel{\text { def }}{=} 9 / 5$ for $\beta_{1} \in[7 / 2,5]$, and all other parameters consistent with the parameter estimations given in Appendix (A). Hence the first correction term is small.

However, the second and higher correction terms need not be negligible for biologically relevant parameters. Noting $B_{(2)}^{0} \in\left[0, \beta_{1}\right]$, the second correction term gives

$$
\begin{aligned}
\left|\frac{\psi_{1}^{2} F^{\prime \prime}\left(\psi_{2}^{0}\right)}{2 F^{\prime 2}\left(\psi_{2}^{0}\right)}\right| & =\frac{\psi_{1}^{2} \rho_{2} \rho_{3} \beta_{1}^{2}\left(\psi_{2}^{0}\right)^{\rho_{3} \beta_{1}-1}}{2\left(1+\left(\psi_{2}^{0}\right)^{\rho_{3} \beta_{1}}\right)^{2}\left|F^{\prime 2}\left(\psi_{2}^{0}\right)\right|} \\
& =\frac{\psi_{1}^{2} \rho_{2} \rho_{3}\left(B_{(2)}^{0}\right)^{1-\frac{1}{\rho_{3} \beta_{1}}}\left(\beta_{1}-B_{(2)}^{0}\right)^{1+\frac{1}{\rho_{3} \beta_{1}}}}{2\left|F^{\prime 2}\left(\psi_{2}^{0}\right)\right|} \lesssim \frac{A^{2} \psi_{1}^{2} \rho_{2} \rho_{3} \beta_{1}^{2}}{2}
\end{aligned}
$$

This indicates that $\psi_{2}=\psi_{2}^{0}$ is a good approximation provided $A^{2} \psi_{1}^{2} \rho_{2} \rho_{3} \beta_{1}^{2} \ll 1$. For higher order terms, simple power counting indicates that the $n^{\text {th }}$ correction term is $O\left(\rho_{2} \rho_{3}^{n-1} \beta_{1}^{n} A^{n} \psi_{1}^{n}\right)$. Consequently, if $A^{2} \psi_{1}^{2} \rho_{3}^{2} \beta_{1}^{2} \ll \min \left(1, \rho_{3} / \rho_{2}\right)$, power counting would subsequently indicate that all correction terms are small and thus that the approximation $\psi_{2}=\psi_{2}^{0}$ is accurate. Note that on varying $\beta_{1}$, with all other parameters as in Table 1 , we always have $\rho_{3} / \rho_{2}<1$, while $A^{2} \psi_{1}^{2} \rho_{2} \rho_{3} \beta_{1}^{2}<\epsilon$ for $\beta_{1}<9$, but $A^{2} \psi_{1}^{2} \rho_{2} \rho_{3} \beta_{1}^{2}>0.75$ for $\beta_{1}>11.5$. This indicates that $\psi_{2}=\psi_{2}^{0}$ is a good approximation for biologically reasonable parameters provided $\beta_{1}$ does 
not lie towards the upper end of its biological bounds. For higher values of $\beta_{1}$, one must solve (51) to obtain a perturbative approximation for $B_{\max }$ which unfortunately does not possess a simpler, but accurate, representation. However note that it is straightforward to deduce from equation (51) that an increase in the value of $\psi_{1}$ always results in a decrease in the value of $B_{\max }$.

\subsubsection{Numerical verification}

Figures 8, 9 and 10 compare the numerical value of $B_{\max }$ to the perturbative approximation given by equation (54), which is represented by the dashed line in all figures, and the perturbative approximation given by the solution of equation (51), which is denoted by the solid line in all figures.

In Figure 8 we consider the effect of varying $\beta_{1}$, fixing all other parameters as given in Table 1. The approximations are excellent for smaller values of $\beta_{1}$. For larger values of $\beta_{1}$, the approximation given by (54) loses accuracy. The solution of (51) yields an improved approximation for larger values of $\beta_{1}$, which is consistent with the above observations.

In Figure 9 we consider the variation of $k_{2}=k_{3}$ in the interval [0.3, 0.83], fixing all other parameters as given in Table 1 . We do not exceed $k_{2}=k_{3}=0.83$, because of the constraint $k_{4}>k_{2}$, as discussed in Appendix A.2. We see that for relatively small $k_{2}=k_{3}$ the estimate given by the solution of (51) is substantially more accurate. Note that while reducing $k_{2}=k_{3}$ does not affect $\psi_{1}$, we have $\rho_{3} \rho_{2} \sim k_{2}^{-1} k_{3}^{-1}$ and hence the condition $\psi_{1}^{2} \rho_{2} \rho_{3} \beta_{1}^{2} \ll 1$ is eventually violated. Consequently, for smaller values of $k_{2}=k_{3}$ one must use the solution of (51). One can observe from Figure 9 that the solution of (51) does indeed yield an estimate for $B_{\max }$ consistent with a term at leading order in $\epsilon$. We note from Figure 8 that

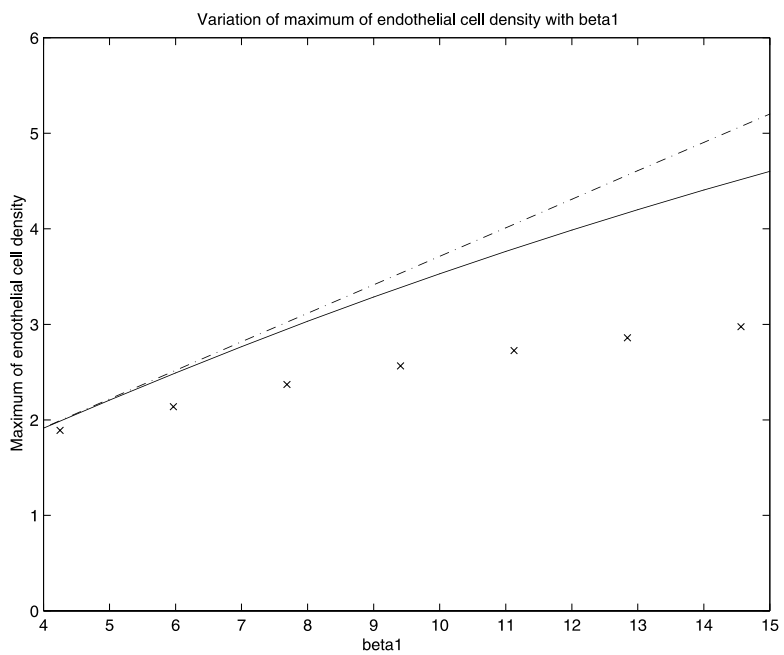

Fig. 8. Comparison of numerical maximum of $B$ (individually plotted) with the approximation given in equation (54) (dashed line) and the solution to equation (51) (solid line) on varying $\beta_{1}$. All other parameters are fixed as in Table 1 


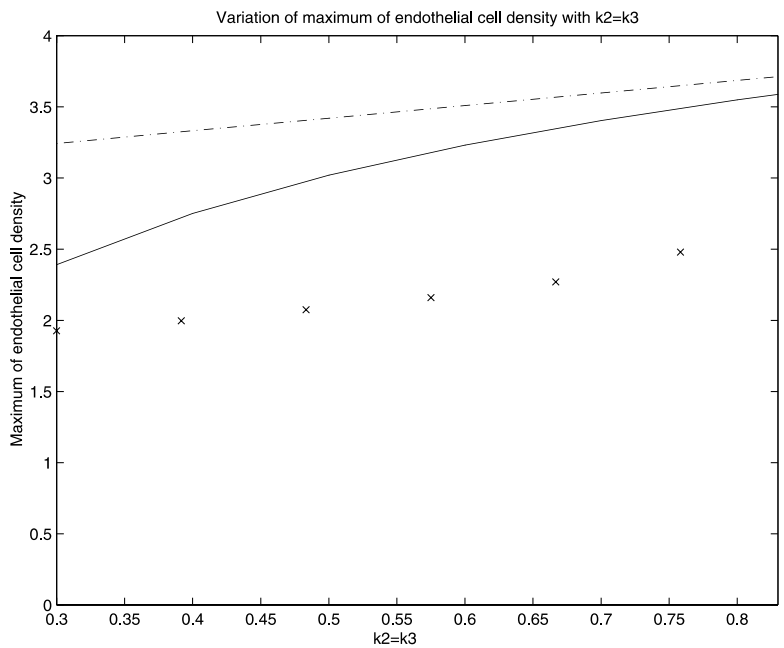

Fig. 9. Comparison of numerical maximum of $B$ (individually plotted) with the approximation given in equation (54) (dashed line) and the solution to equation (51) (solid line) on varying $k_{2}=k_{3}$. All parameters are fixed as in Table 1, except $k_{2}=k_{3}$, which are varied in the interval $[0.3,0.83]$

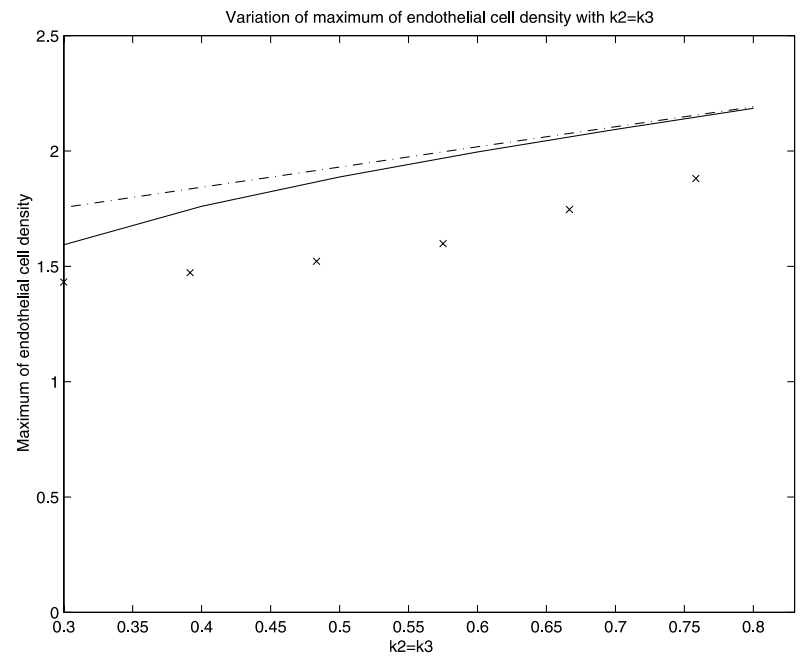

Fig. 10. Comparison of numerical maximum of $B$ (individually plotted) with the approximation given in equation (54) (dashed line) and the solution to equation (51) (solid line) on varying $k_{2}=k_{3}$. All parameters are fixed as in Table 1 , except that $\beta_{1}=5$ and $k_{2}=k_{3}$ are varied in the interval $[0.3,0.83]$

the analytical approximations for $B_{\max }$ are more accurate for smaller $\beta_{1}$; in figure 10 , we see further evidence of this, where we consider the variation of $k_{2}=k_{3}$ in the interval [0.3, 0.83], but now with $\beta_{1}=5.0$, while fixing all other parameters as given in Table 1. Obviously, one should also consider the variation of $B_{\max }$ with 
$k_{5}$; this is addressed below, as part of an investigation as to whether amending cell motility parameters can affect the angiogenic response.

\section{Discussion}

\subsection{Qualitative predictions and the propagation Speed}

By consideration of the main cellular processes involved in wound-healing angiogenesis, we have developed and analysed a new model for wound-healing angiogenesis. It differs from previous models in that it focuses only on the endothelial cells and the capillary tips, thus constituting a minimal model for which analytical insight can, and should, be developed, before extension and generalisation.

All our simulations of the model show the development of a wave-like profile progressing towards the centre of the wound, exhibiting the qualitative features empirically observed in cutaneous wound healing. There is a definite 'structural unit', consisting of a peak of capillary tips, closely followed by a peak in blood vessel density, moving in concert towards the wound centre. Additionally, the whole wounded region exhibits elevated endothelial cell density, as one would expect with the formation of granulation tissue in the wound. Thus the model's qualitative predictions are in complete accord with observation. Consequently, one is interested in the quantitative predictions of the model, and how these may be interpreted in terms of a healing wound.

The first quantitative prediction of this model is that the speed of the structural unit is given by the theoretical minimum travelling wave speed, $\sqrt{4 C_{1} k_{2}}$. Numerically, one always observes that the actual speed of the structural unit asymptotically tends to $\sqrt{4 C_{1} k_{2}}$ on the biological domain. One must note that the validity of this observation is not necessarily obvious given the model does not evolve to a travelling wave on the biological domain. Nonetheless, the minimum travelling wave speed has proven to be a very accurate predictor for the structural unit's speed of propagation in the model. Thus, the structural unit's speed is determined simply by the rate $C_{1}$ of capillary tip diffusive random motion and the rate $k_{2}$ of tip branching. We note that a stochastic model of angiogenesis has produced a similar prediction; that the rate of vessel ingrowth is strongly dependent on the rate of endothelial cell migration, but not on the rate of proliferation (as long as some takes place) $[15,18]$.

\subsection{Predictions for the capillary tip density maximum}

Despite the relative complexity of this multiparameter model, one can apply perturbative techniques to obtain information concerning travelling wave solutions, which in turn, give useful information concerning the structural unit. One can determine that a crude approximation for the maximum of the capillary tip density, is given by

$$
\begin{aligned}
N_{\text {max }} & =\frac{k_{2}}{k_{3}}\left(1-\rho_{2} \beta_{1} \psi_{*}^{\rho_{3} \beta_{1}}\right) \\
\psi_{*}^{\rho_{3} \beta_{1}} & =\left(\frac{\rho_{3} \beta_{1}+1}{\rho_{2} \rho_{3} \beta_{1}^{2}}\left(\frac{k_{5} \rho_{4}}{\rho_{3} \beta_{1}} \epsilon^{5}\right)^{\frac{1}{\rho_{3} \beta_{1}}}\right)^{\frac{\rho_{3} \beta_{1}}{\rho_{3} \beta_{1}+1}},
\end{aligned}
$$


and this has explicitly been shown to agree, at least approximately, with the numerical simulations for a wide range of parameter values. This agreement is useful for two reasons. Firstly, it lends support to our hypothesis that one can determine useful information concerning the structural unit from the minimum speed travelling wave. Secondly, it should be noted that increasing capillary tip densities within the structural unit relies primarily upon increasing $k_{2} / k_{3}$, and on reducing $\rho_{2} \beta_{1} \psi_{*}^{\rho_{3} \beta_{1}}$. The fact that $k_{2} / k_{3}$ strongly influences $N_{\max }$ is not surprising; however, the fact that the other parameters influence $N_{\max }$ by the parameter grouping $\rho_{2} \beta_{1} \psi_{*}^{\rho_{3} \beta_{1}}$ would be difficult to predict other than by analytical means.

\subsection{Predictions for the endothelial cell density maximum}

Detailed consideration of $N_{\max }$ is not as relevant or interesting as investigating the maximum of the endothelial cell density, $B_{\max }$, within the structural unit, as this constitutes a measure of the angiogenic ability of the system and its ability to supply nutrients to the wound healing structural unit. First of all, the perturbative framework enables us to make the modelling prediction that altering aspects of the endothelial cell density remodelling mechanism will not increase the strength of the angiogenic response. This is because the terms governing this mechanism within the model are pre-multiplied by $\rho_{4} \epsilon^{4}$ so, increasing the effects of this mechanism by a factor of 50 will not change the perturbative structure of the model. Furthermore, if $\beta_{1}$ is small, so that $\psi_{1}^{2} \rho_{3}^{2} \beta_{1}^{2} \ll \min \left(1, \rho_{3} / \rho_{2}\right)$, upregulating the remodelling mechanism, corresponding to altering parameters so as to increase $\rho_{4}$, simply will not affect the angiogenic response. If the parameters however are such that $\psi_{1}$ is not negligible, then increasing $\rho_{4}$ will increase $\psi_{1}$, which will lead to a counter intuitive decrease in $B_{\text {max }}$. However, this reduction will be relatively small as the dependence of $B_{\text {max }}$ on $\rho_{4}$ is via $\psi_{1} \sim \rho_{4}^{\left(\rho_{3} \beta_{1}\right)^{-1}}$, and thus is weak due to the fact that $\rho_{3} \beta_{1}$ is large when $\psi_{1}^{2} \rho_{3}^{2} \beta_{1}^{2} \ll \min \left(1, \rho_{3} / \rho_{2}\right)$. Thus, we have the prediction that increasing the remodelling response will, at best, have no effect or, at worst, be mildly counter-productive.

In Figure 11 we test this observation numerically by fixing all parameters, except $v$, as in Table 1 , and observing that increasing $v$ by a factor of 50 results only in a small decrease of $B_{\max }$, as predicted above.

Looking at how $B_{\max }$ depends on the model parameters in the perturbative framework indicates that $B_{\max }$ is independent of the parameters influencing cell motility, except possibly via the $k_{5}$ dependence of $\psi_{1}$. There are a number of points to note here. Firstly, $k_{5}$ is a ratio of carrying capacities, and hence one may anticipate that is is not easily manipulated experimentally. Secondly, it is difficult to estimate $k_{5}$. Thirdly, we have the scaling $\psi_{1} \sim\left(k_{5} v \epsilon^{5}\right)^{1 / \rho_{3} \beta_{1}}$ and the observation, from Figure 11, that while varying $v$ by a factor of 50 does alter $\psi_{1}$, it is only enough to cause small changes in $B_{\text {max }}$. Consequently, varying $k_{5}$ over one, possibly even two, orders of magnitude again only results in small changes in $B_{\max }$. Thus, we have following conclusions. Firstly, our inability to estimate $k_{5}$ accurately does not affect our ability to make predictions for $B_{\max }$. Secondly, we have the modelling prediction that the angiogenic response of the system, as measured by $B_{\max }$ is, at 


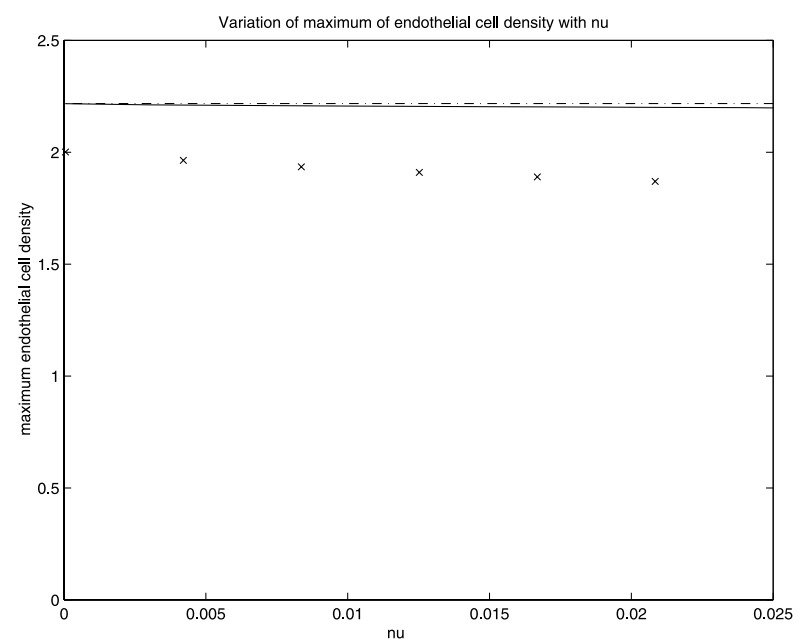

Fig. 11. Comparison of numerical maximum of $B$ (individually plotted) with the approximation given in equation (54) (dashed line) and the solution to equation (51) (solid line) on varying $v$ in the interval $[0.0005,0.025]$. All other parameter values are as in Table (1, except that $\beta=5.0$. The graph for $\beta=10.0$, not shown, is very similar, though the asymptotic approximations are less accurate, as observed in section (4.6.3)

leading orders of magnitude, insensitive to parameters influencing cell motility, including $k_{5}$ which only weakly influences $B_{\max }$, via $\psi_{1}$. Such predictions have been validated against simulations of the full partial differential equation model. For example, in Figure 12, we consider the variation in $k_{5}$, which, as one would anticipate from the asymptotics, yields results which are very similar to those presented in Figure 11. One is primarily interested in increasing the angiogenic capabilities of the system and thus $B_{\max }$, which depends on the parameters $\beta_{1}, \rho_{2} \beta_{1}, \rho_{3} \beta_{1}$ and $\psi_{1}$. However, as we have seen in all figures the general trend of how $B_{\max }$ depends on parameter values is reproduced by equation (54), with the influence of $\psi_{1}$ acting as a corrective term for higher values of $\beta_{1}$. To determine how one may manipulate parameters so as to induce as large an increase as possible in $B_{\max }$ we therefore focus on equation (54), and hence the parameters $\beta_{1}, \rho_{2}$ and $\rho_{3}$.

The parameter $\beta$ represents the ratio of the carrying capacity of capillary tip induced endothelial cell proliferation to the carrying capacity of logistic remodelling endothelial cell proliferation. Consequently, one would intuitively expect that increasing $\beta$ will increase $B_{\max }$, the (non-dimensionalised) endothelial cell density, as observed in Figure 8. However, it is likely that manipulation of $\beta$ is not empirically possible. Hence we treat $\beta$ as fixed. Furthermore, we have $\beta_{1}=\beta+k_{5} k_{4} / \chi$. We consider below only variations induced by manipulating the angiogenic system such that

$$
\delta\left(\frac{k_{5} k_{4}}{\chi}\right) \ll \beta_{1}=\beta+\frac{k_{5} k_{4}}{\chi} .
$$




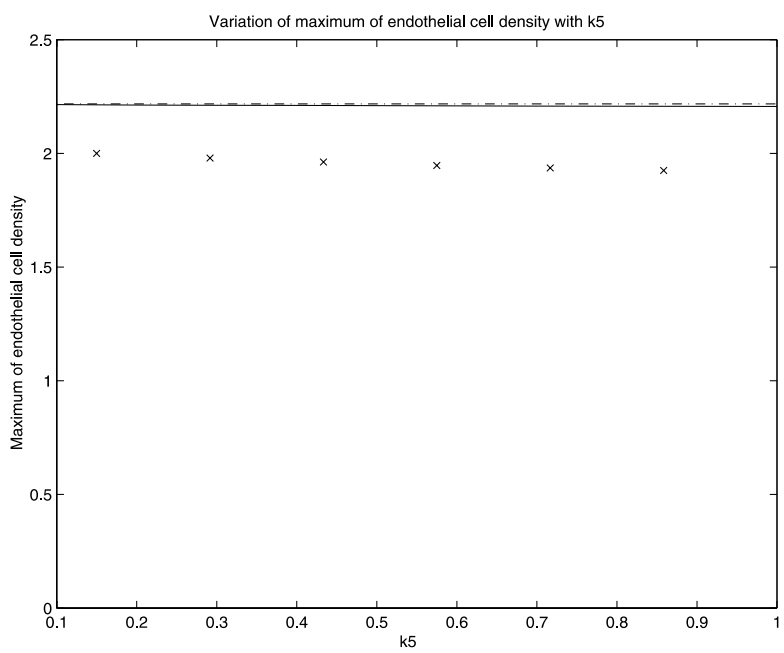

Fig. 12. Comparison of numerical maximum of $B$ (individually plotted) with the approximation given in equation (54) (dashed line) and the solution to equation (51) (solid line) on varying $k_{5}$ in the interval $[0.1,1.0]$. All other parameter values are as in Table (1, except that $\beta=5.0$. The graph for $\beta=10.0$, not shown, is very similar, though the asymptotic approximations are less accurate, as observed in section (4.6.3)

i.e. that $\beta_{1}$ is essentially unchanged by the variation of $k_{5} k_{4} / \chi$. Note that $k_{5}$ is a function of carrying capacities, and is therefore unlikely to be altered by empirical manipulations of the angiogenesis system. Given that $k_{5} k_{4} / \chi \ll \beta$ for biologically reasonable parameters, this restriction is therefore that the empirical manipulations of the angiogenesis system do not induce changes in $\left(k_{4} / \chi\right)$ that are orders of magnitude greater than typically measured values of $\left(k_{4} / \chi\right)$. With this restriction, we are consequently interested in variations of

$$
\rho_{2}=\frac{k_{4}}{k_{2}}, \quad \rho_{3}=\frac{\chi}{k_{3}}
$$

where $k_{4}$ governs the strength of anastomosis, $k_{2}$ governs the proliferation rate of capillary tips, $k_{2}=k_{3}$ and $\chi$ governs the rate of capillary tip induced endothelial cell proliferation.

It is straightforward to deduce from equation (54) that $\psi_{2}^{0}$, and hence $B_{(2)}^{0} \sim$ $B_{\text {max }}$, is monotonically increasing in $\rho_{3}$ and monotonically decreasing in $\rho_{2}$. This is in complete accord with intuition. For example, it predicts that stimulating capillary tip induced endothelial cell proliferation increases $B_{(2)}^{0} \sim B_{\max }$, whereas stimulating anastomosis decreases $B_{(2)}^{0} \sim B_{\text {max }}$.

Given an angiogenic scenario, one is interested in what specific changes can be made so as to maximally increase blood vessel formation. Typically, one can alter cell motility and cell proliferation rates via the stimulation of growth factor production, or the exogenous addition of growth factors. Clearly, the model predicts that adding factors that simply stimulate cell motility will not greatly affect blood vessel formation. Adding growth factors will typically reduce cell cycle times but will 
not affect carrying capacities, which generally are determined by other limitations, such as requirements for nutrients and physical space. Consider adding growth factor which uniformly stimulates proliferation for all cell types (without affecting carrying capacities). One would have the scaling $k_{2} \rightarrow \Phi k_{2}, k_{3} \rightarrow \Phi k_{3}, \chi \rightarrow \Phi \chi$ and $v \rightarrow \Phi v$, with $\Phi>1$, which will decrease $\rho_{2}$ but will leave $\rho_{3}$ unaffected. Consider instead, adding a cell specific factor that preferentially stimulates capillary tip proliferation, but again does not affect carrying capacities. This will again decrease $\rho_{2}$ but it will also decrease $\rho_{3}$, and consequently cannot give a greater increase in blood vessel formation than simply increasing cell proliferation uniformly. Consider a third possibility, whereby one adds a cell specific factor that preferentially stimulates cells other than those constituting the capillary tips, and again does not affect carrying capacities. This will increase $\chi$ (and $v$ ) while leaving $k_{2}$ and $k_{3}$ unaffected, and so will increase $\rho_{3}$, and not affect $\rho_{2}$. This leaves the interesting possibility that stimulating all cells to proliferate may be less effective in increasing blood vessel formation than stimulating all cells except those at capillary tips. One clearly has the requirement that $B_{\max }$ is more sensitive to changes in $\rho_{2}$ than $\rho_{3}$ for the stimulation of all cells to proliferate is more effective.

However, the relative sensitivities of $B_{\max }$ to the parameters $\rho_{2}$ and $\rho_{3}$ is not immediately obvious, even when considering the perturbative approximations for $B_{\text {max }}$. We consider the simplest approximation, namely $B_{(2)}^{0}$, as given by equation (54), since it is relatively easy to manipulate and yet, as we have seen above on numerous occasions, successfully predicts the general dependence of $B_{\max }$ on model parameters. Working with this approximation, one can show, after some algebra, that

$$
\Psi \stackrel{\text { def }}{=}\left|\frac{\partial B_{(2)}^{0} / \partial \rho_{2}}{\partial B_{(2)}^{0} / \partial \rho_{3}}\right|=\left[\rho_{2}^{2} \beta_{1}^{2}\left(\psi_{2}^{0}\right)^{\rho_{3} \beta_{1}} \int_{-\infty}^{0} d z \frac{z e^{\left(\rho_{3} \beta_{1}+1\right) z}}{\left(1+\left(\psi_{2}^{0}\right)^{\rho_{3} \beta_{1}} e^{\rho_{3} \beta_{1} z}\right)^{2}}\right]^{-1} .
$$

For $\left(\psi_{2}^{0}\right)^{\rho_{3} \beta_{1}} \ll 1$, one can readily approximate the integral to determine that

$$
\Psi_{\text {approx }} \sim \frac{\left(\rho_{3} \beta_{1}+1\right)^{2}}{\rho_{2}^{2} \beta_{1}^{2}\left(\psi_{2}^{0}\right)^{\rho_{3} \beta_{1}}}
$$

and, via numerical integration, one can confirm that $\Psi=\Psi_{\text {approx }}$ holds to a within a relative error of $\sim 35 \%$ for $\left(\psi_{2}^{0}\right)^{\rho_{3} \beta_{1}}<1$.

Therefore, when

$$
\Psi_{\text {approx }}<1 \quad \text { and } \quad\left(\psi_{2}^{0}\right)^{\rho_{3} \beta_{1}}<1
$$

one has a leading order prediction that $B_{(2)}^{0}$ is more sensitive to the parameter $\rho_{3}$ than $\rho_{2}$. Consequently one has the leading order prediction that targeting proliferative stimulation of cells other than those at capillary tips would, under such circumstances, be more effective than unspecific proliferative stimulation. We proceed to verify this with the improved approximation to $B_{\max }$, given by the solution of equation (51). 

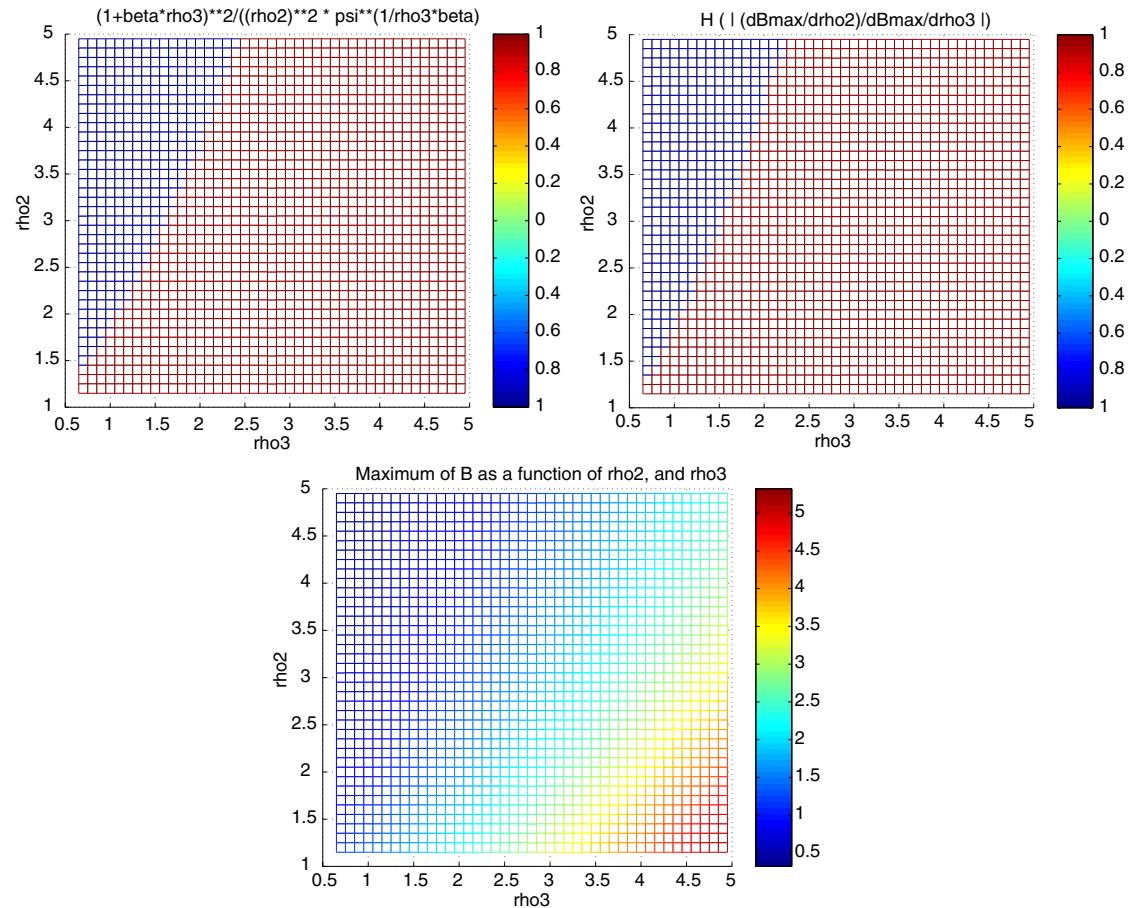

Fig. 13. In these graphs, $\beta_{1}=5.5$ and $\rho_{2}, \rho_{3}$ are varied. For the upper left graph we plot $\left.H\left(\left(\rho_{3} \beta_{1}+1\right)^{2}\right) /\left(\rho_{2}^{2} \beta_{1}^{2}\left(\psi_{2}^{0}\right)^{\rho_{3} \beta_{1}}\right)\right)$, where the function $H$ is given in equation (62). For the upper right graph, we plot $H\left(\left|\left(\partial B_{(2)} / \partial \rho_{2}\right) /\left(\partial B_{(2)} / \partial \rho_{3}\right)\right|\right)$, where $B_{(2)} \sim B_{\max }$ is calculated via the improved perturbative approximation, equation (51). $B_{(2)}^{0}$ is plotted on the lower graph. Note the similarity between the upper left and right graphs, and that the region (in blue) where $H=-1$ in these graphs corresponds to regions where $B_{(2)}^{0}<\beta_{1} / 2=2.75$ on the right-hand graph, i.e. $\left(\psi_{2}^{0}\right)^{\rho_{3} \beta_{1}}<1$. See text for further details

In all graphs of Figure 13, $\beta_{1}=5.5$, while the parameters $\rho_{2}$ and $\rho_{3}$ are varied. In the upper left hand graph of Figure 13 we plot the function

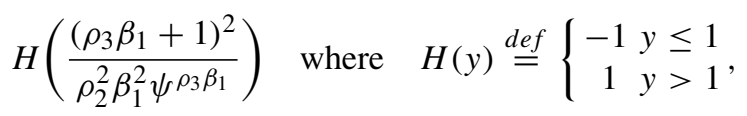

while in the upper right graph we plot

$$
H\left(\left|\frac{\partial B_{(2)} / \partial \rho_{2}}{\partial B_{(2)} / \partial \rho_{3}}\right|\right)
$$

where $B_{(2)} \sim B_{\text {max }}$ and its derivatives are calculated via the improved perturbative approximation, equation (51). One can see that there is a good correlation between the approximation arising from the use of $\Psi_{\text {approx }}$ and from equation (51) as to the regions of parameter space where $B_{\max }$ is more sensitive to changes in $\rho_{3}$. Consider the lower graph of Figure 13, which is a plot of $B_{(2)}^{0}$. One can see that the region 
where $B_{(2)}^{0}$ is predicted to be more sensitive to $\rho_{3}$ (in blue in the upper graphs of Figure (13)) are regions where $B_{(2)}^{0}<\beta_{1} / 2(=2.75)$, i.e. $\left(\psi_{2}^{0}\right)^{\rho_{3} \beta_{1}}<1$. Hence, as required, the second of the conditions in equation (61) holds in this region.

Consequently, we have a modelling prediction that the region of parameter space where preferential proliferative stimulation of cells excluding those at capillary tips induces a greater angiogenic response is characterised by ${ }^{1}$

$$
\begin{aligned}
1>\Psi_{\text {approx }} & =\frac{\left(\rho_{3} \beta_{1}+1\right)^{2}}{\rho_{2}^{2} \beta_{1}^{2} \psi^{\rho_{3} \beta_{1}}}=\frac{\rho_{3}^{2}}{\rho_{2}^{2} \psi^{\rho_{3} \beta_{1}}}\left(1+\frac{1}{\beta_{1}}\right)^{2}=\frac{\chi^{2} k_{2}^{2}}{k_{3}^{2} k_{4}^{2} \psi^{\rho_{3} \beta_{1}}}\left(1+\frac{1}{\beta_{1}}\right)^{2} \\
& =\frac{\chi^{2}}{k_{4}^{2} \psi^{\rho_{3} \beta_{1}}}\left(1+\frac{1}{\beta_{1}}\right)^{2}
\end{aligned}
$$

Furthermore, for $\psi^{\rho_{3} \beta_{1}} \ll 1$ one has, from equation (54), $\psi^{\rho_{3} \beta_{1}} \sim \rho_{3} / \rho_{2} \sim$ $\chi / k_{4}$. Noting that $\beta_{1}^{-1}$ is small, the above condition can be refined to

$$
\psi^{\rho_{3} \beta_{1}} \ll 1, \text { or } 1>\frac{\chi^{2}}{k_{4}^{2}} \text { for } \psi^{\rho_{3} \beta_{1}} \sim 1 .
$$

Consequently, we can summarise the above by making the modelling prediction that, subject to the weak restriction given by equation (57), the preferential proliferative stimulation of cells excluding those at capillary tips induces a greater angiogenic response in scenarios characterised by one of the following. There is either an extremely low level of angiogenic response $\left(\psi^{\rho_{3} \beta_{1}} \ll 1\right)$ or there is a low angiogenic response $\left(\psi^{\rho_{3} \beta_{1}} \leq \sim 1\right)$ coupled with high levels of anastomosis $\left(k_{4}\right)$ and/or low levels of proliferation in the vicinity of capillary tips $(\chi)$.

Acknowledgements. It is a pleasure to acknowledge useful advice from Professor Jonathan Sherratt concerning this work. Part of this work was undertaken while EAG was funded by The Wellcome Trust, Grant No 047521. KP acknowledges support from a EPSRC Pool Studentship.

\section{A. Appendix. Non-dimensionalisation and parameter estimation}

\section{A.1. Dimensional model}

The dimensional model is

$$
\frac{\partial n}{\partial t}=\frac{\partial}{\partial x}\left[D_{1} \frac{\partial n}{\partial x}\right]+\frac{\partial}{\partial x}\left[D_{2} n\right]+\lambda_{1} n+\lambda_{2} n-\lambda_{3} n^{2}-\lambda_{4} n b
$$

${ }^{1}$ One may consider increasing $\beta_{1}$ : the analogous graphs to those presented in Figure 13, but with $\beta_{1}=10$ (not shown), illustrate that the trends depicted in Figure 13 are unchanged, though the correlation between the analogues of the upper left and the upper right graphs of Figure 13 is slightly weaker. This is not surprising from the fact that $B_{(2)}$, given in equation (54), becomes a less accurate predictor for the solution of (51) as $\beta_{1}$ increases (see section (4.6.2) and Figure 8). Similarly, one would also expect that the correlation between $\Psi$ and $\left|\left(\partial B_{\max } / \partial \rho_{2}\right) /\left(\partial B_{\max } / \partial \rho_{3}\right)\right|$ will decrease in accuracy as $\beta_{1}$ increases, but will nonetheless predict the correct trends. 


$$
\begin{gathered}
\frac{\partial b}{\partial t}=\lambda_{5} \frac{\partial}{\partial x}\left[D_{1} \frac{\partial n}{\partial x}+D_{2} n \frac{\partial b}{\partial x}\right]+\lambda_{6} \chi n b\left(b_{1}-b\right) \\
+\lambda_{6} v b\left(b_{0}-b\right)+\lambda_{5}\left(\lambda_{3} n^{2}+\lambda_{4} n b\right) \\
n(x, 0)= \begin{cases}0 & x \in\left[0, x_{0}\right) \\
n_{\text {init }} & x=x_{0}\end{cases} \\
b(x, 0)= \begin{cases}0 & x \in\left[0, x_{0}\right) \\
b_{0} & x=x_{0}\end{cases}
\end{gathered}
$$

We nondimensionalise the model using the following scalings for the variables:

$$
\tilde{n}=\frac{n}{n_{0}}, \quad \tilde{b}=\frac{b}{b_{0}}, \quad \tilde{t}=\frac{t}{T}, \quad \tilde{x}=\frac{x}{x_{0}} .
$$

Dimensionless parameters are defined as follows:

$$
C_{1}=\frac{D_{1} T}{x_{0}^{2}}, \quad C_{2}=\frac{D_{2} T b_{0}}{x_{0}^{2}}, k_{2}=\lambda_{2} T, \quad k_{3}=\lambda_{3} n_{0} T,
$$

$k_{4}=\lambda_{4} b_{0} T, \quad k_{5}=\frac{\lambda_{5} n_{0}}{b_{0}}, \quad \tilde{\chi}=\lambda_{6} T b_{1} n_{0} \chi, \quad \tilde{v}=\lambda_{6} T b_{0} \nu, \quad \beta=\frac{b_{1}}{b_{0}}, \quad \tilde{\varsigma}=\frac{\varsigma}{x_{0}}$,

$\widetilde{n_{\text {init }}}=\frac{n_{\text {init }}}{n_{0}}$

This enables one to write down the non-dimensionalised equations, which are given at the end of section (2), where tildes are dropped for convenience.

\section{A.2. Parameter Estimates}

In the following, the estimation of $n_{0}, x_{0}, T$ and the non-dimensionalised parameters listed in (64) is discussed in detail. The results of the section are summarised in Table 1.

The value $x_{0}$ used in the non-dimensionalisation is the half-width of the wound and taken to be $0.5 \mathrm{~cm} . T$ is taken to be 1 day $=86400$ seconds.

Without loss of generality, one may conveniently define $n_{0}$ to be the dimensionalised carrying capacity of capillary tips. To the authors' knowledge it is not possible to estimate this carrying capacity from empirical observation as there is currently no experimental data for capillary tip densities. However, it serves only one purpose in the model, namely to scale from the non-dimensionalised model predictions of capillary tip density to give dimensionalised predictions. Thus, as long as we need only consider relative, rather than absolute, values of capillary tip and endothelial cell densities for our modelling observations and conclusions, such restrictions do not affect the modelling. Note that with this definition of $n_{0}$ we require $k_{2}=k_{3}$.

Variations of $\tilde{\zeta}$ and $\widetilde{n_{\text {init }}}$ do not affect the model greatly, in that they determine initial conditions, which are "forgotten" as the solution moves towards its travelling wave attractor (provided the initial conditions still lie in the basin of the 
travelling wave attractor). We take $\tilde{\zeta}$ and $\widetilde{n_{\text {init }}}$ to be small (relative to unity) with typical values of 0.25 . This simply represents the fact that the preliminary elevated capillary tip density does not extend extensively into the unwounded area, and does not exceed $n_{\max }$ at $t=0$. The latter can be verified $a$ posteriori, by consideration of Figure 2 for example. The diffusion coefficient associated with individual and independent cells is typically between $10^{-9}$ and $10^{-8} \mathrm{~cm}^{2} \mathrm{~s}^{-1}$ [6]. The lower bound is particularly appropriate for this case, as the motion of the endothelial cells constituting the capillary tips involves the orchestrated movement of a number of cells in unison, which may reduce the effective diffusion coefficient, possibly below even $10^{-9} \mathrm{~cm}^{2} \mathrm{~s}^{-1}$. Taking $D_{1}=10^{-9} \mathrm{~cm}^{2} \mathrm{~s}^{-1}$ yields $C_{1}=3.5 \times 10^{-4}$ for the non-dimensionalised diffusion coefficient (though this may be an overestimate). We note that $C_{1}$ is the key diffusion coefficient, as together with $k_{2}$, it dictates the travelling wave speed (see section (4.1)). We estimate $C_{2}$ simply by $C_{1}=C_{2}$ so that neither of the capillary tip transport mechanisms, i.e. random motion and convection down endothelial cell gradients, dominate in the model.

The parameter grouping $\lambda_{6} v b_{0}$ can be estimated by considering the normal slow remodelling of the vasculature, with $n=0$. Ignoring spatial terms, and at low endothelial cell densities, we have approximately $d b / d t \sim \lambda_{6} v b_{0} b$. On average $\lambda_{6} v b_{0} b$ new cells are created per unit length per unit time. In a study [7] of endothelial cell proliferation in various normal and cancerous tissues, tritated thymidine injections were used to label proliferating cells, differentiating endothelial cells from other cell types by morphology. In normal skin it was found that the number of labelled cells per hundred endothelial cells increased by about 0.25 per day over seven days. Thus, by considering a one day time interval, we have

$$
\tilde{v}=\lambda_{6} \nu b_{0} T \sim \ln \left(\frac{100+0.25}{100}\right) \sim \frac{1}{400}
$$

giving an estimate for $\tilde{v}$. We are, of course, ignoring cell cycle subtleties by using the number of cells entering S-phase in any given time period as an estimate for the number of cells that would have entered M-phase. However, such numbers are of the same order of magnitude, and hence the methodology is legitimate for order of magnitude estimates.

The parameter $\tilde{\chi}$ can be estimated by considering the maximum endothelial cell proliferation rate permitted by the model. For $b$ small, and $n$ relatively large we have

$$
\frac{d b}{d t} \sim \lambda_{6}\left(v b_{0}+n_{\max } \chi b_{1}\right) b .
$$

The same study [7] also included a study of endothelial cell proliferation in near optimal conditions during placental growth. It was found that within two days of beginning the tritiated thymidine injections $79.5 \%$ of endothelial cells were labelled. Thus we have the estimate

$$
\tilde{\chi} \frac{n_{*}}{n_{0}} \sim \chi \lambda_{6} n_{*} \chi b_{1} T \sim \frac{1}{2} \ln (1.8)-\tilde{v} \sim \frac{1}{2} \ln (1.8)
$$


where $n_{*}$ denotes the capillary tip density attained under optimal conditions, which is the carrying capacity density assuming capillary tip loss due to anastomosis is minimal. We thus take $n_{*} \sim n_{0}$ giving an estimate that

$$
\tilde{\chi} \sim \frac{1}{2} \ln (1.8) \sim 0.3 .
$$

Dyson et al. [5] used both cell counts and microangiography combined with computer-assisted image analysis to measure vascularization of porcine dermal wounds in moist and dry environments. Using endothelial cell counts, it was found that the maximum ratio of the number of endothelial cells per unit area in the wound space (mean \pm standard deviation) compared to the number in uninjured dermis was $6.7 \pm 1.8$ for a moist wound environment, and $4.5 \pm 1.5$ for a dry wound environment. The corresponding results using microangiography were 14.3 from vessel counts and 5.0 from percentage area measurements. These give an estimate for $\beta$ in the approximate range $[3.5,15]$. We initially work with $\beta$ in the middle of this range, taking $\beta=9.29$ (with the 3SF accuracy specified so that the ubiquitous parameter $\beta_{1} \stackrel{\text { def }}{=} \beta+k_{5} k_{4} / \chi$ is equal to 10.0 , a convenient round number, for the reference set of parameters).

The parameter $k_{2}$ can be estimated from the doubling time of capillary tips. This is estimated via the typical doubling time of a proliferating cell (20-24 hours), as capillary tip proliferation is ultimately driven by endothelial cell proliferation. Thus, we take $k_{2}=(24 / 20) \ln 2 \sim 0.8$, which gives us $k_{3}$. The parameter $k_{4}$ is more difficult to estimate. We now motivate the constraint $k_{4} \geq k_{2}$. One should consider the travelling wave connecting the wounded steady-state $(n, b)=(0,0)$ to the unwounded steady-state $(n, b)=(0,1)$ in the phase-plane. The equation for $n$ decouples from $b$ in a linear expansion about these steady-states, giving

$$
\begin{aligned}
C_{1} n^{\prime \prime}-c n^{\prime}+k_{2} n=0 & & (n, b) \sim(0,0) \\
C_{1} n^{\prime \prime}-c n^{\prime}+k_{2} n-k_{4} n=0 & & (n, b) \sim(0,1) .
\end{aligned}
$$

Given $c, k_{4}>0$, and with $c^{2} \geq 4 C_{1} k_{2}$ as required for non-negativity near $(0,0)$, the eigenvalues at $(0,0)$ and $(0,1)$ are all of the same sign for $k_{4}<k_{2}$, making a connection between the two steady-states impossible. Thus, one must take $k_{4} \geq k_{2}$ for a connection between the above two steady-states. We briefly note that with $k_{4}<k_{2}$ another non-negative steady-state exists, and with $k_{4}=k_{2}$, this steady-state confluences with the steady-state $(n, b)=(0,1)$. Consequently, for $k_{4} \leq k_{2}$ there is a bifurcation in phase space. We do not consider this in the present paper. Further work is required to investigate whether it represents a mathematical artefact, or a bifurcation yielding travelling waves linking $(0,0)$ to a different, possibly pathological, steady-state. We therefore take $k_{4}>k_{2}$ in this paper. Furthermore, if $k_{4}$ is excessively larger than $k_{2}$, the model would be likely to be trivial with no capillary tip growth owing to excessive tip-sprout anastomosis, and thus $k_{4}$ and $k_{2}$ should be of the same order of magnitude. Consequently, we initially take $k_{4}=0.85$.

Estimating $k_{5}$ is difficult; all we can do is present a upper bound for $k_{5}$. Recalling that the parameter $\lambda_{5}$ is the number of endothelial cells within a capillary tip, 
the parameter $k_{5} \stackrel{\text { def }}{=} \lambda_{5} n_{0} / b_{0}$ represents the ratio of endothelial cells at the carrying capacity limits of capillary tip density and remodelled endothelial cell density. During the formation of the granular tissue only a minority of the endothelial cells should arise indirectly, via capillary tip proliferation and subsequent anastomosis for the following reason. If anastomosis and other means of endothelial cell proliferation or migration introduced cells into localised endothelial cell compartments at similar rates one would produce a blood vessel network with comparable densities of blood vessel junctions and endothelial cells, which would be inconsistent with typical blood vessel networks. The bulk of the formation of the structural unit within the granular tissue in the model occurs in the inner region described in section (4.3), as minimal angiogenesis occurs in the wavefront region, while the waveback region is predominantly governed by the remodelling process. Consequently, one anticipates that in the inner region the increase in endothelial cell density due to anastomosis is subleading compared to the increase in endothelial cell density due to other mechanisms in the model, the dominant one of which is proliferation. With the definitions $\rho_{3}=\tilde{\chi} / k_{3}$ and $\beta_{1}=\beta+k_{5} k_{4} / \tilde{\chi}$, this requires that $k_{5} /\left(\rho_{3} \beta_{1}\right)$ in equation (27) is asymptotically small, giving a bound for $k_{5}$. Thus we only consider values of $k_{5}$ for which $k_{5} / \rho_{3} \beta \leq O(\epsilon)$, where $\epsilon(=1 / 4)$ is the asymptotic parameter in our perturbative expansion. This reasoning underlies the derivation of equation (28). For definiteness, we take $k_{5}=1 / 4$ in Table 1 which, of course, satisfies the above constraint.

\section{B. Numerics}

The equations of this model constitute a set of non-linear coupled with convective-diffusion equations, with non-linear kinetics. A minor point to note is that, in practice, the initial conditions are slightly smoothed to facilitate numerical solution. The key difficulty with numerically solving these equations is the presence of diffusion and convection simultaneously, which tends to yield artefact oscillations in many numerical schemes. Computer speed is not particularly important with one-dimensional simulations, and hence we used an explicit, rather than an implicit, method. Artefact numerical oscillations are avoided by use of upwinding. This, however, introduces the possibility of excessive artefactual numerical diffusion. This can typically be avoided in two ways. Use of a sufficiently small mesh, if possible, is adequate; otherwise, one can resort to recovery methods. It proved possible to use an explicit scheme, with upwinding, and a sufficiently small mesh to avoid numerical artefact diffusion for this model. Further details on these, and other, numerical techniques for convection-diffusion equations can be found in [9].

\section{Error bounds in perturbation theory}

We consider the first correction term in section (4.6.2), with parameter values such that $\beta_{1}>5$, in which case $\rho_{3} \beta_{1}>5 / 3$ and $\rho_{2} \beta_{1}>5$. These constitute all but extreme values of the parameter space outlined in Appendix (A.2). Let

$$
K\left(\psi, \rho_{3} \beta_{1}, b\right)=\int_{0}^{\psi} \frac{d x}{1+x^{\rho_{3} \beta_{1}}}-\frac{\psi}{1+\psi^{\rho_{3} \beta_{1}}}-\frac{1}{b}\left(\frac{\psi \psi^{\rho_{3} \beta_{1}}}{1+\psi^{\rho_{3} \beta_{1}}}\right)
$$


with $\psi \geq 0$ and $b \geq 2$, where $b$ should be considered a parameter, which can, if required, be adjusted to fine tune any bound deduced below. We have that $K\left(0, \rho_{3} \beta_{1}, b\right)=0$ and

$$
\frac{\partial K}{\partial \psi}=\frac{\psi^{\rho_{3} \beta_{1}}}{b\left(1+(\psi)^{\rho_{3} \beta_{1}}\right)}\left(\rho_{3} \beta_{1}(b-1)-1-\psi^{\rho_{3} \beta_{1}}\right) .
$$

Hence $\partial K / \partial \psi>0$ for any sufficiently small $\psi>0$; thus $K\left(\psi, \rho_{3} \beta_{1}, b\right)>0$ for $\psi \in\left(0, \psi_{*}\right]$ where $\psi_{*}$ is the value of $\psi$ at the only turning point for $\psi>0$, and is given by $\psi_{*}^{\rho_{3} \beta_{1}}=\rho_{3} \beta_{1}(b-1)-1$. Noting that

$$
\frac{1}{\psi_{2}^{0}} \int_{0}^{\psi_{2}^{0}} \frac{d x}{1+x^{\rho_{3} \beta_{1}}}=\frac{\rho_{2} \beta_{1}-1}{\rho_{2} \beta_{1}}
$$

we have

$$
\begin{aligned}
K\left(\psi_{2}^{0}, \rho_{3} \beta_{1}, b\right)= & \frac{\psi_{2}^{0}}{\rho_{2} \beta_{1}\left(1+\left(\psi_{2}^{0}\right)^{\rho_{3} \beta_{1}}\right)}\left(\rho_{2} \beta_{1}\left(1-\frac{1}{b}\right)-1\right) \\
& \times\left[\left(\psi_{2}^{0}\right)^{\rho_{3} \beta_{1}}-\frac{1}{\rho_{2} \beta_{1}\left(1-\frac{1}{b}\right)-1}\right] .
\end{aligned}
$$

Note that we have

$$
\left(\rho_{2} \beta_{1}\left(1-\frac{1}{b}\right)-1\right)>0
$$

Set $b=2$. Suppose $\left(\psi_{2}^{0}\right)^{\rho_{3} \beta_{1}} \leq\left(\rho_{2} \beta_{1}\left(1-\frac{1}{b}\right)-1\right)^{-1}$.

Then $K\left(\psi_{2}^{0}, \rho_{3} \beta_{1}, b\right) \leq 0$. Hence one must have $\psi_{2}^{0}>\psi_{*}$, i.e. $\left(\psi_{2}^{0}\right)^{\rho_{3} \beta_{1}}>$ $\left(\rho_{3} \beta_{1}(b-1)-1\right)$. This gives an immediate contradiction if $\rho_{3} \beta_{1}(b-1)-1>$ $\left(\rho_{2} \beta_{1}\left(1-b^{-1}\right)-1\right)^{-1}$, i.e. if

$$
\left(\rho_{3} \beta_{1}(b-1)-1\right)\left(\rho_{2} \beta_{1}\left(1-\frac{1}{b}\right)-1\right)-1>0 .
$$

It is a straightforward matter to check that, given $\rho_{3} \beta_{1}>5 / 3, \rho_{2} \beta_{1}>5$, taking $b=2$ is sufficient to enforce the above inequality. Hence for $b=2$ we have, by contradiction, $\left(\psi_{2}^{0}\right)^{\rho_{3} \beta_{1}}>\left(\rho_{2} \beta_{1}\left(1-\frac{1}{b}\right)-1\right)^{-1}$.

Recall that

$$
\begin{aligned}
F(p) & =\left(1-\rho_{2} \beta_{1}\right) p+\rho_{2} \beta_{1} \int_{0}^{p} \frac{d x}{1+x^{\rho_{3} \beta_{1}}}, \\
\psi_{1} & =\left(B_{(1)} /\left(\beta_{1}-B_{(1)}\right)\right)^{1 /\left(\rho_{3} \beta_{1}\right)}=\left(\frac{k_{5} \rho_{4}}{\rho_{3} \beta_{1}} \epsilon^{5}\right)^{\frac{1}{\rho_{3} \beta_{1}}}\left(1+O\left(\frac{\epsilon}{\rho_{3} \beta_{1}}\right)\right) \ll 1
\end{aligned}
$$


A straightforward rearrangement of the inequality $\left(\psi_{2}^{0}\right)^{\rho_{3} \beta_{1}}>\left(\rho_{2} \beta_{1}\left(1-b^{-1}\right)^{-1}\right.$ $-1)^{-1}$ yields

$$
\left|F^{\prime}\left(\psi_{2}^{0}\right)\right|=\frac{\rho_{2} \beta_{1}\left(\psi_{2}^{0}\right)^{\rho_{3} \beta_{1}}}{1+\left(\psi_{2}^{0}\right)^{\rho_{3} \beta_{1}}}-1>\frac{1}{b-1}
$$

and hence, with $\beta_{1}>5$ and thus $b=2$, one has

$$
\left|\frac{\psi_{1}}{F^{\prime}\left(\psi_{2}^{0}\right)}\right|<\psi_{1} \text {. }
$$

It is straightforward to use the same technique with $b=14 / 5$ and $\beta_{1} \in[7 / 2,5]$ and hence $\rho_{3} \beta_{1}>5 / 4$ and $\rho_{2} \beta_{1}>7 / 2$. The resulting bound is valid for any parameter values consistent with the estimation in Appendix (A) above. For $b=14 / 5$ one can deduce that $\left(\psi_{2}^{0}\right)^{\rho_{3} \beta_{1}}>\left(\rho_{2} \beta_{1}\left(1-\frac{1}{b}\right)-1\right)^{-1}$ and thus

$$
\left|\frac{\psi_{1}}{F^{\prime}\left(\psi_{2}^{0}\right)}\right|<9 / 5 \psi_{1} \text {. }
$$

when $\beta_{1} \in[7 / 2,5]$. Inequalities (70) and (71) give the required bounds for section (4.6.2).

\section{References}

[1] Arnold, F., West, D.C.: Angiogenesis in wound healing. Pharmac. Ther., 52, 407-422 (1991)

[2] Byrne, H.M., Chaplain, M.A.J., Hopkinson, I., Evans, D.: Mathematical modelling of angiogenesis in wound healing: comparison of theory and experiment. J. Theor. Medicine, In Press, 1999

[3] Clark, R.A.F.: Overview of wound repair. In R. A. F. Clark, editor, The Molecular and Cellular Biology of Wound Repair, chapter 1. Plenum, second edition, 1996

[4] Maini, P.K., Dallon, J.C., Sherratt, J.A.: Mathematical modelling of extracellular matrix dynamics using discrete cells: Fiber orientation and tissue. J. Theoretical Biology, 199(4), 449-471 (1999)

[5] Dyson, M., Young, S.R., Hart, J., Lynch, J.A.: The effects of moist and dry conditions on angiogenesis. Journal of Investigative Dermatology, 1992

[6] Gaffney, E.A., Maini, P.K., McCaig, C.D., Zhao, M., Forrester, J.: Modelling corneal epithelial wound closure in the presence of physiological electric fields via a moving boundary formalism. IMA J. Mathematics Applied in Medicine and Biology, 16(4), 369-393 (1999)

[7] Hobson, B., Denekamp, J.: Endothelial proliferation in tumours and normal tissues : Continuous labelling studies. British Journal of Cancer, 49, 405-413 (1984)

[8] Olsen, L., Sherratt, J.A., Maini, P.K.: A mathematical model for fibro-proliferative wound healing disorders. B. Math. Biology, 58, 787-807 (1996)

[9] Morton, K.: Numerical Solution of Convection-Diffusion Problems. Chapman \& Hall, first edition, 1996

[10] Olsen, L., Sherrat, J.A., Maini, P.K., Arnold, F.: A mathematical model for the capillary endothelial cell-extracellular matrix interactions in wound-healing angiogenesis. IMA Journal of Mathematics Applied in Medicine and Biology, 14(4), 261-281 (1997) 
[11] Sherratt, J., Dale, P., Maini, P.: Mathematical modelling corneal epithelial wound healing. Mathematical Biosciences, 127, 124 (1994)

[12] Maini, P., Dale, P., Sherratt, J.: On the speed of corneal epithelial wound healing. Appl. Math. Lett., 7, 11-14 (1994)

[13] Pettet, G.J., Byrne, H.M., McElwain, D.L., Norbury, J.: A model of wound healing angiogenesis in soft tissue. Math. Biosci., 136, 35-63 (1996a)

[14] Pettet, G.J., Chaplain, M.A.J., McElwain, D.L., Byrne, H.M.: On the role of angiogenesis in wound healing. Proc. Roy. Soc. Ser. B, 263, 1487-1493 (1996b)

[15] Stokes, C.L.: Endothelial cell migration and chemotaxis in angiogenesis. In R. Steiner, P. B. Weisz, and R. Langer, editors, Angiogenesis: Key Principles-Science-Technology-Medicine, 118-124 Birkhäuser Verlag, 1992

[16] Williams, P.L., Warwick, R., Dyson, M., Bannister, L.H.: Gray's Anatomy. Churchill Livingstone, 37th edition, 1989

[17] Bennett, N.T., Schultz, G.S.: Growth Factors and Wound Healing II. Role in normal and chronic wound healing, Am. J. Surgery, 166, 74-81 1993

[18] Anderson, S., Chaplain, M.A.J.: Continuous and discrete mathematical models of tumour induced angiogenesis, Bull Math Bio, 60, 857-899 (1998)

[19] Balding, D., McElwain, D.L., A Mathematical-Model of Tumor-Induced Capillary Growth J. Theoretical Biology, 114, 53-73 (1985)

[20] Edelstein, L.: The propagation of fungal colonies - a model for tissue-growth $J$. Theoretical Biology, 98, 679-701 (1982) 